\title{
Hopfological algebra for infinite dimensional Hopf algebras
}

\author{
Marco A. Farinati * \\ June 5, 2019
}

\begin{abstract}
2010 Mathematics Subject Classification: 16T05 16E35, 18G99, 18D99, 19A49, 81R50 Keywords: Co-Frobenius Hopf Algebras, Tensor Triangulated Categories, Homology Theories, $K_{0}$, Categorification.
\end{abstract}

\begin{abstract}
We consider "Hopfological" techniques as in $[\mathrm{K}]$ but for infinite dimensional Hopf algebras, under the assumption of being co-Frobenius. In particular, $H=$ $k[\mathbb{Z}] \# k[x] / x^{2}$ is the first example, whose corepresentations category is d.g. vector spaces. Motivated by this example we define the "Homology functor" (we prove it is homological) for any co-Frobenius algebra, with coefficients in $H$-comodules, that recover usual homology of a complex when $H=k[\mathbb{Z}] \# k[x] / x^{2}$. Another easy example of co-Frobenius Hopf algebra gives the category of "mixed complexes" and we see (by computing an example) that this homology theory differs from cyclic homology, although there exists a long exact sequence analogous to the SBIsequence. Finally, because we have a tensor triangulated category, its $K_{0}$ is a ring, and we prove a "last part of a localization exact sequence" for $K_{0}$ that allows us to compute -or describe- $K_{0}$ of some family of examples, giving light of what kind of rings can be categorified using this techniques.
\end{abstract}

\section{Introduction}

This paper has mainly 3 contributions:

(1) The "Hopfological algebra" can be developed not only for finite dimensional Hopf algebras but also for infinite dimensional ones, provided they are co-Frobenius. The language of comodules is better addapted than the language of modules.

(2) The formula "Im $d /$ Ker $d$ " can written in Hopf-co-Frobenius language.

(3) Some $K$-theoretical results allow us to compute $K_{0}$ of the stable categories associated to co-Frobenius Hopf algebras of the form $H_{0} \# \mathfrak{B}$, with $H_{0}$ cosemisimple and $\mathfrak{B}$ finite dimensional.

*Partially supported by UBACyT 2018-2021, 256BA. Research member of CONICET - I.M.A.S. Depto. de Matemática, F.C.E.y N. Universidad de Buenos Aires, Ciudad Universitaria Pabellón I (1428) C.A.B.A. Argentina, E-mail address: mfarinatedm. uba . ar 
The paper is organized as follows: In Section 1 we exhibit point (1) above, in Section 2 we make point (2). In Section 3 we develop some tools to understand the triangulated structure. In Section 4 we exhibit the first examples. Section 5 deals with $K_{0}$. Section 6 illustrate the first step on how to develop -in the setting of co-Frobenius Hopf algebrasthe direction taken in [Qi1] for finite dimensional Hopf algebras.

\section{Acknowledgments}

I wish to thank Juan Cuadra for answering questions and pointing useful references on co-Frobenius coalgebras. I also thanks Gastón A. García for helping me with coradical problems. This work was partially supported by UBACyT 2018-2021, 256BA.

\section{Integrals, Co-Frobenius and triangulated structure}

$k$ will be a field, $H$ a Hopf algebra over $k$, all comodules are right comodules. The category of comodules is denoted $\mathcal{M}^{H}$ and the subcategory of finite dimensional comodules is denoted $\mathfrak{m}^{H}$.

\subsection{Integrals}

Definition 1.1. (Hochschild, 1965; G. I. Kac, 1961; Larson-Sweedler, 1969). A (left) integral is a linear map $\Lambda: H \rightarrow k$ such that

$$
(\mathrm{id} \otimes \Lambda) \Delta h=\Lambda(h) 1 \forall h \in H
$$

that is, $h_{1} \Lambda\left(h_{2}\right)=\Lambda(h) 1$.

It is well-known that the dimension of the space of (left) integral is $\leq 1$. In case $H$ admits a non-zero (left) integral $\Lambda \in H^{*}, H$ will be called co-Frobenius. The following is well-known, we refer to [ACE] and [AC] and references therein for the proofs:

Theorem 1.2. If $H$ is co-Frobenius then, in the category of (say right) $H$-comodules

1. there exists enough projectives;

2. every finite dimensional comodule is a quotient of a finite dimensional projective, and embeds into a finite dimensional injective;

3. being projective is the same as being injective.

If $M$ and $N$ are two objects in a category $\mathcal{B}$, denote $\operatorname{Hom}_{\mathcal{B}}(M, N)$ set of morphisms and $I_{\mathcal{B}}(M, N)$ the subset of $\operatorname{Hom}_{\mathcal{B}}(M, N)$ consisting on morphisms that factors through an injective object of $\mathcal{B}$. Denote

$$
\underline{\operatorname{Hom}}_{\mathcal{B}}(M, N):=\frac{\operatorname{Hom}_{\mathcal{B}}(M, N)}{I_{\mathcal{B}}(M, N)}
$$

The category whose objects are $H$-comodules and morphism $\underline{\operatorname{Hom}}^{H}$ is called the stable category and it is denoted $\underline{\mathcal{M}}^{H}$. Similarly $\underline{\mathfrak{m}}^{H}$ is the stable category associated to $\mathfrak{m}^{H}$. By the above theorem, $\underline{\mathfrak{m}}^{H}$ is embedded fully faithfully in $\underline{\mathcal{M}}^{H}$. With these preliminaries, one can prove the following main construction: 
Theorem 1.3. If $H$ is a co-Frobenius Hopf algebra then $\underline{\mathcal{M}}^{H}$ has a natural structure of triangulated category, $\underline{\mathfrak{m}}^{H}$ is a triangulated subcategory.

Proof. We apply directly Happel's Theorem 2.6 of [Ha]. The only thing to do is to notice that $\mathcal{M}^{H}$ (and $\mathfrak{m}^{H}$ ) are Frobenius exact categories. Using Happel's notation, let $\mathcal{B}$ be an additive category embedded as a full and extension-closed subcategory in some abelian category $\mathcal{A}$, and $\mathcal{S}$ the set of short exact sequences in $\mathcal{A}$ with terms in $\mathcal{B}$. For as, since both $\mathcal{M}^{H}$ and $\mathfrak{m}^{H}$ are already abelian, we have $\mathcal{A}=\mathcal{B}$ and the notion of $\mathcal{S}$-projective and $\mathcal{S}$-injective is the same as usual projectives and injectives. Maybe we just remark that an object in $\mathfrak{m}^{H}$ is injective in $\mathfrak{m}^{H}$ if and only if it is injective in $\mathcal{M}^{H}$ and similarly for projectives (see Lemma 1.4 as an illustration).

An exact category $(\mathcal{B}, \mathcal{S})$ is called a Frobenius category if $(\mathcal{B}, \mathcal{S})$ has enough $\mathcal{S}$ projectives and enough $\mathcal{S}$-injectives and if moreover the $\mathcal{S}$-projectives coincide with the $\mathcal{S}$-injectives. In our case, $\mathcal{B}=\mathcal{M}^{H}$ or $\mathcal{B}=\mathfrak{m}^{H}$ are clearly Frobenius categories if $H$ is a co-Frobenius Hopf algebra. Theorem 2.6 in [Ha] just state that the stable category $\underline{\mathcal{B}}$ is triangulated.

Lemma 1.4. If $P \in \mathfrak{m}^{H}$ then $P$ is projective in $\mathfrak{m}^{H}$ if and only if it is projective in $\mathcal{M}^{H}$.

Proof. If $P$ is projective in $\mathcal{M}^{H}$ then then it has the lifting property for all comodules, in particular for the finite dimensional ones. Assume $P$ is projective in $\mathfrak{m}^{H}$ and consider a diagram of comodules

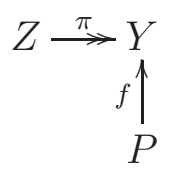

where $Z$ and $Y$ are not necessarily finite dimensional. Since $P$ is finite dimensional, one can consider $Y^{\prime}=f(P) \subseteq Y$, clearly $Y^{\prime}$ is a finite dimensional comodule, with generators say $\left\{y_{1}, \ldots, y_{n}\right\}$. Since $\pi$ is surjective, one may found $z_{i}(i=1, \ldots, n)$ with $\pi\left(z_{i}\right)=y_{i}$ and there exists a finite dimensional subcomodule $Z^{\prime} \subseteq Z$ containing all $z_{i}{ }^{\prime} \mathrm{s}$, hence we have a diagram

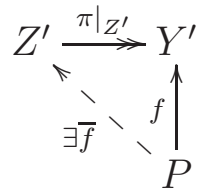

Now all comodules are finite dimensional, and because $P$ is projective within finite dimensional comodules, there exists a lifting $\bar{f}: P \rightarrow Z^{\prime} \subseteq Z$ of $f$, hence, a lifting of the original $f$.

For clarity of the exposition we recall the definition of suspension, desuspension and triangles in $\underline{\mathcal{M}}^{H}$. For this particular case of comodules over a co-Frobenius Hopf algebra, the general definitions can be more explicitly realized. Moreover, for $H=$ $H_{0} \# \mathfrak{B}$ as in Section 3, concrete and functorial constructions can be done in $\mathfrak{m}^{H}$. The reader familiar with Happel's results may go directly to Section 2. 


\subsection{Suspension and desuspension functors}

In $[\underline{\mathrm{K}}]$, when $H$ is finite dimensional and $\Lambda$ is an integral in $H$ (not in $H^{*}$ ), the author embeds an $H$-module $X$ via $X \otimes \Lambda \subset X \otimes H$ and define $T(X)$ as $(X \otimes H) /(X \otimes \Lambda)$. For us, $\Lambda \in H^{*}$ and this definition makes no sense, but (even without using the integral) one can always embed an $H$-comodule $M$ into $M \otimes H$ by means of its structural map. The structure map $\rho$ is $H$-colinear provided we use the (co)free $H$-comodule structure on $M \otimes H$ (and not the diagonal one).

Definition 1.5. For a right $H$ comodule $M$ with structure $\rho: M \rightarrow M \otimes H$, define

$$
T(M):=(M \otimes H) / \rho(M)
$$

If $H$ is finite dimensional this definition also makes sense in $\mathfrak{m}^{H}$. If $H$ is co-Frobenius and $0 \neq M$ is finite dimensional, $(M \otimes H) / \rho(M)$ is not finite dimensional, however, there exists a finite dimensional injective $I(M)$ and a monomorphism $M \rightarrow I(M)$, so, one can define $I(M) / M$ in $\underline{\mathfrak{m}}^{H}$ and we know $T(M) \cong I(M) / M$ in $\underline{\mathcal{M}}^{H}$. Moreover, for co-Frobenius Hopf algebras, one can give functorial embeddings $M \rightarrow I(M)$ in $\mathcal{M}^{H}$ that works in $\mathfrak{m}^{H}$ (see Corollary 2.16).

Remark 1.6. If the notation $M \otimes H$ is confusing because $H$ is Hopf and one also has the diagonal action, one may consider another injecting embedding:

$$
\begin{gathered}
i_{M}: M \rightarrow M \otimes H \\
m \mapsto m \otimes 1
\end{gathered}
$$

This map is clearly an embedding, and it is $H$-colinear if one uses the diagonal action on $M \otimes H$. Both embeddings are ok because $M \otimes H$ with diagonal action and $M \otimes H$ with structure coming only from $H$ are isomorphic (see Lemma 2.14).

Similar (or dually) to $[\underline{K}]$ one can define desuspension. Consider the map $\Lambda^{\prime}=\Lambda \circ S$

$$
\Lambda^{\prime}: H \rightarrow k
$$

Recall that $H$ co-Frobenius implies $S$ is bijective (see for instance [DNR]) and it is easy to prove that $\Lambda^{\prime}=\Lambda \circ S$ is a right integral:

$$
\begin{gathered}
\Lambda^{\prime}\left(h_{1}\right) h_{2}=\Lambda\left(S\left(h_{1}\right)\right) h_{2}=\Lambda\left(S\left(h_{1}\right)\right) S^{-1} S\left(h_{2}\right) \\
\left.\left.\left.=S^{-1}\left(\Lambda\left(S\left(h_{1}\right)\right) S\left(h_{2}\right)\right)=S^{-1}\left(\Lambda\left(S(h)_{2}\right)\right) S(h)_{1}\right)\right)=S^{-1}(\Lambda(S h) 1)\right)=\Lambda(S h) 1=\Lambda^{\prime}(h) 1
\end{gathered}
$$

We use $\Lambda^{\prime}$ because $\Lambda^{\prime}$ is right colinear:

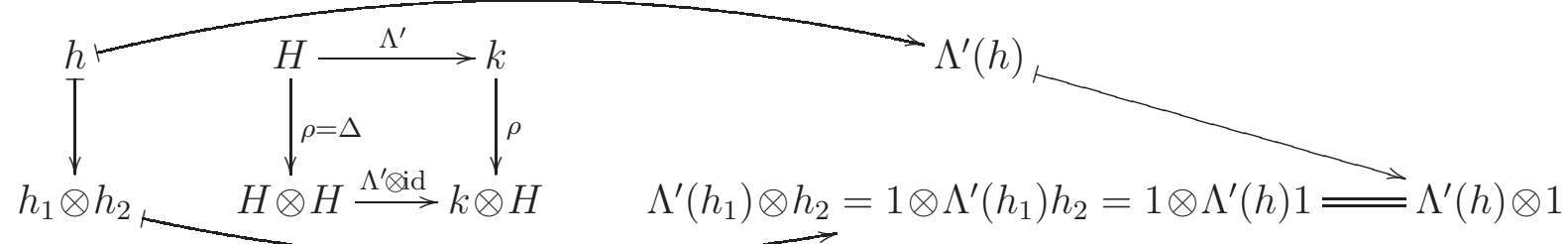

Hence, $\operatorname{Ker}\left(\Lambda^{\prime}\right)$ is a right $H$-comodule and we have, for any $M$, a short exact sequence

$$
0 \rightarrow M \otimes \operatorname{Ker}\left(\Lambda^{\prime}\right) \rightarrow M \otimes H \rightarrow M \rightarrow 0
$$


Definition 1.7. The desuspension functor is $T^{\prime}(M):=M \otimes \operatorname{Ker}\left(\Lambda^{\prime}\right) \in \underline{\mathcal{M}}^{H}$

Remark 1.8. When considering $\mathfrak{m}^{H}$, we know every finite dimensional comodule $M$ has a finite dimensional projective cover $P(M) \rightarrow M$, so we can consider $T^{\prime \prime}(M):=$ $\operatorname{Ker}(P(M) \rightarrow M) \in \underline{\mathfrak{m}}^{H}$ and this is isomorphic to $T^{\prime}(M)$ in the stable category. Also (see Corollary 2.16), one can define $P(M)$ in $\mathcal{M}^{H}$ and in $\mathfrak{m}^{H}$ in a functorial way.

As an illustration of the need of stabilization for having a triangulated category one see that, for any $M$ a comodule, we have a short exact sequence in $\mathcal{M}^{H}$

$$
0 \rightarrow M \rightarrow M \otimes H \rightarrow T M \rightarrow 0
$$

In particular, considering $T^{\prime} M$ instead of $M$, there is a short exact sequence

$$
0 \rightarrow T^{\prime} M \rightarrow T^{\prime} M \otimes H \rightarrow T T^{\prime} M \rightarrow 0
$$

But there is also a short exact sequence

$$
0 \rightarrow T^{\prime} M \rightarrow M \otimes H \rightarrow M \rightarrow 0
$$

So " $M$ computes $T T^{\prime} M$ using another injective embedding". Usually $T T^{\prime} M \nsubseteq M$ in $\mathcal{M}^{H}$ but $M \cong T T^{\prime} M$ in $\underline{\mathcal{M}}^{H}$. Similar argument for $T^{\prime} T$, hence these are mutually inverse functors in the stable category, but not in $\mathcal{M}^{H}$.

\subsection{Triangles}

One of the axioms of triangulated categories is that any map $f: X \rightarrow Y$ is a part of a triangle $X \stackrel{f}{\rightarrow} Y \rightarrow Z \rightarrow T X \rightarrow$. Triangles are defined via the mapping cone construction. For $f: X \rightarrow Y, C o(f)$ is defined in the following way:

Choose an injective embedding $i: X \rightarrow I(X)$ and define $C o(f)$ by the square

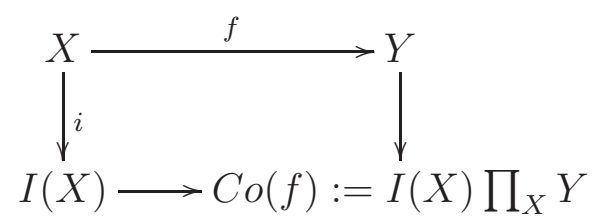

One can see that this definition does not depend -in the stable category- on the choice of the injective embedding $X \rightarrow I(X)$. Notice also a well defined map $C o(f) \rightarrow T(X)$ given by the universal property of the push-out:

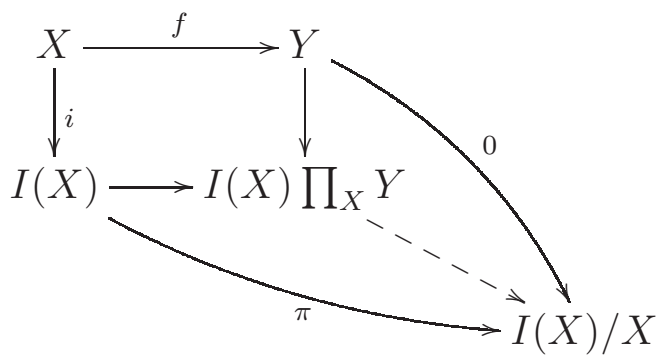

Triangles $X \rightarrow Y \rightarrow Z \rightarrow T X$ in $\underline{\mathcal{M}}^{H}$ are (by definition) all sequences isomorphic (in $\underline{\mathcal{M}}^{H}$ ) to some sequence of the form $X \stackrel{f}{\rightarrow} Y \rightarrow C o(f) \rightarrow T(X)$. Next two Lemmas emphasize the strong relation between the exact structure of $\mathcal{M}^{H}\left(\right.$ resp $\left.\mathfrak{m}^{H}\right)$ and the triangulated structure of $\underline{\mathcal{M}}^{H}\left(\operatorname{resp} \underline{\mathfrak{m}}^{H}\right)$ 
Lemma 1.9. If $0 \rightarrow X \stackrel{u}{\rightarrow} Y \stackrel{\pi}{\rightarrow} Z \rightarrow 0$ is a short exact sequence in $\mathcal{M}^{H}$ then the sequence $X \rightarrow Y \rightarrow Z$ is isomorphic to $X \rightarrow Y \rightarrow C o(u)$ in the stable category.

Proof. We assume $Z=Y / u(X)$. Consider the diagram

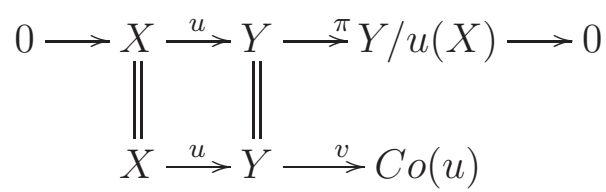

Let $X \rightarrow I(X)$ be an embedding into an injective object, for simplicity we assume $X \subseteq I(X)$. We define the map

$$
\begin{aligned}
I(X) \oplus Y & \longrightarrow Z \\
(e, y) & \mapsto \pi(y)
\end{aligned}
$$

It has the property that, for any $x \in X$,

$$
(-x, u(x)) \mapsto \pi(u(x))=0
$$

So, it induces a well defined map

$$
\begin{gathered}
C o(u)=\frac{I(X) \oplus Y}{(x, 0) \sim(0, u(x))} \longrightarrow Z \\
\overline{(e, y)} \mapsto \pi(y)
\end{gathered}
$$

Now from the injectivity of $I(X)$ we know there exists a map fitting into the diagram

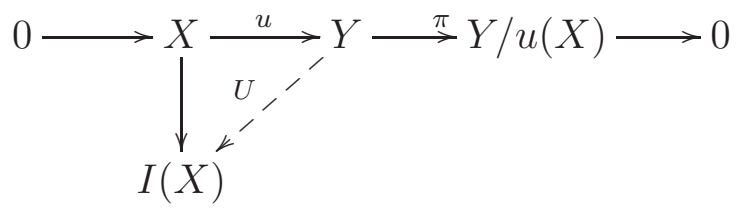

So, define the map

$$
\begin{gathered}
Y \rightarrow C o(u) \\
y \mapsto \overline{(U(y), y)}
\end{gathered}
$$

It has the property

$$
u(x) \mapsto \overline{(-U(u(x)), u(x))}=\overline{(-x, u(x))}=0
$$

so, it induces a well defined map

$$
Z=Y / u(X) \rightarrow C o(u)
$$

One composition is the identity:

$$
\begin{gathered}
Z \rightarrow C o(u) \rightarrow Z \\
z=\pi(y) \mapsto \overline{(-U(y), y)} \mapsto \pi(y)=z
\end{gathered}
$$


The other composition is

$$
\begin{gathered}
C o(u) \rightarrow Z \rightarrow C o(u) \\
\overline{(e, y)} \mapsto \pi(y) \mapsto \overline{(-U(y), y)}
\end{gathered}
$$

so, the Kernel is

$$
\{\overline{(e, y)}: y \in u(X)\} \cong \frac{I(X) \oplus u(X)}{(x, 0) \sim(0, u(x))} \cong I(X)
$$

that is an injective comodule, so, these morphisms are mutually inverses in $\underline{\mathcal{M}}^{H}$.

The second Lemma is a useful one, maybe it is folklore but it is not usually written: Lemma 1.10. If $X \rightarrow Y \rightarrow Z \rightarrow T X$ is a triangle in the stable category then there exists a short exact sequence $0 \rightarrow X^{\prime} \rightarrow Y^{\prime} \rightarrow Z^{\prime} \rightarrow 0$ in $\mathcal{M}^{H}$ such that the sequence $X \rightarrow Y \rightarrow Z$ is isomorphic to $X^{\prime} \rightarrow Y^{\prime} \rightarrow Z^{\prime}$ in the stable category.

Proof. One of the axioms of triangulated categories says that $X \rightarrow Y \rightarrow Z \rightarrow T X$ is a triangle if and only if $T^{-1} Z \rightarrow X \rightarrow Y \rightarrow Z$ is so. Hence, $T^{-1} Z \rightarrow X \rightarrow Y \rightarrow Z$ is isomorphic to a distinguished triangle, that is, there is an isomorphism (in the stable category) of t-uples

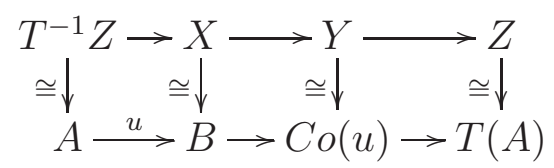

In particular, there is a commutative diagram in the stable category

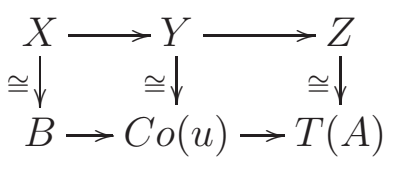

and clearly $0 \rightarrow B \rightarrow C o(u) \rightarrow T(A) \rightarrow 0$-or equivalently

$$
0 \rightarrow B \rightarrow I(A) \prod_{A} B \rightarrow I(A) / A \rightarrow 0,
$$

is a short exact sequence in $\mathcal{M}^{H}$. Notice that if $A$ and $B$ are finite dimensional, one can find a finite dimensional injective hull $I(A)$ and hence the short exact sequence also belongs to $\mathfrak{m}^{H}$.

\section{Integrals and coinvariants}

If $C$ is a coalgebra and $M$ a right $C$-comodule then $M$ is a left $C^{*}$ module via

$$
\phi \cdot m:=\phi\left(m_{1}\right) m_{0} \quad\left(\phi \in C^{*}, m \in M\right)
$$

where, as usual, if $M$ is a right $H$-comodule, we denote $\rho: M \rightarrow M \otimes H$ its structural map and we use Seedler-type notation $\rho(m)=m_{0} \otimes m_{1} \in M \otimes H$. In particular, for $C=H$ and $\phi=\Lambda \in H^{*}$, being left integral means $\Lambda \cdot h=\Lambda(h) 1$. Moreover, multiplication by $\Lambda$ in $M$ has the following standard and main property:

$\rho(\Lambda \cdot m)=\rho\left(\Lambda\left(m_{1}\right) m_{0}\right)=\Lambda\left(m_{2}\right) m_{0} \otimes m_{1}=m_{0} \otimes \Lambda\left(m_{2}\right) m_{1}=m_{0} \otimes \Lambda\left(m_{1}\right) 1=(\Lambda \cdot m) \otimes 1$

That is, $\Lambda \cdot M \subseteq M^{c o H}$. We list some examples, keeping in mind the above formula. 


\section{Examples}

1. If $H$ is co-semisimple (e.g. $H=\mathcal{O}(G)$ with $G$ an affine reductive group) then the inclusion $k \rightarrow H$ split as $H$-comodules. One can check that an $H$-colinear splitting is an integral. In the cosemisimple case, the inclusion $\Lambda \cdot M \subseteq M^{c o H}$ is actually an equality (this will be clear in Subsection 2.1). Nevertheless, the integral may not be so explicitly described. An easy and explicit example is:

2. If $G$ is a finite group and $H=k^{G}$, then $\Lambda=\sum_{g \in G} g \in k[G] \cong\left(k^{G}\right)^{*}$ is an integral. For any $f \in k^{G}$ :

$$
\Lambda(f)=\sum_{g \in G} f(g)
$$

Actually, every finite dimensional Hopf algebra is (Frobenius and) co-Frobenius. Notice that $k^{G}$ is co-semisimple if and only if $k[G]$ is semisimple, if and only if the characteristic of the ground field does not divide the order of $G$.

3. Let $G$ be a group (possibly infinite, e.g. $G=\mathbb{Z}$ ) and $H=k[G]$, define

$$
\Lambda\left(\sum_{g \in G} \lambda_{g} g\right):=\lambda_{1_{G}}
$$

A right $H$-comodule $M$ is the same as $G$-graded vector space $M=\oplus_{g \in G} M_{g}$. The action of $\Lambda$ gives the projection into $M_{1_{G}}$.

4. Tensor product of co-Frobenius algebras is co-Frobenius, the integral can be computed using tensor products of integrals.

5. Let $H$ be a Hopf algebra and $H_{0}$ its coradical. Notice that $H_{0}$ does not need to be a Hopf subalgebra in general. Nevertheless, one of the main results in [ACE] is that $H$ is co-Frobenius if and only if the coradical filtration is finite. A particular case is illustrated in the following:

6. Let $H_{0}$ be a cosemisimple Hopf algebra and let $V \in{ }_{H_{0}} \mathcal{Y} \mathcal{D}^{H_{0}}$ be a finite dimensional Yetter-Drinfel'd module such that its Nichols algebra $\mathfrak{B}=\mathfrak{B}(V)$ is finite dimensional. Then $H=H_{0} \# \mathfrak{B}$ is co-Frobenius. The integral is essentially given by the "volume form", or "Fermionic integration" in $\mathfrak{B}$ (see Remark 3.5).

(a) The simplest example is: $H$ generated by $x$ and $g^{ \pm 1}$ with relations $x^{2}=0$ and $g x=-x g$. Comultiplication given by

$$
\begin{gathered}
\Delta g=g \otimes g \\
\Delta x=x \otimes g+1 \otimes x
\end{gathered}
$$

The antipode is

$$
S(g)=g^{-1}, S(x)=-x g^{-1}=g^{-1} x
$$

We have $H \cong k[\mathbb{Z}] \# k[x] / x^{2}$. An element of $h$ may be uniquely written as

$$
h=\sum_{n \in \mathbb{Z}} a_{n} g^{n}+\sum_{n \in \mathbb{Z}} b_{n} g^{n} x \quad\left(a_{n}, b_{n} \in k\right)
$$


A left integral is given by

$$
\Lambda(h):=b_{0}
$$

This particular example motivates all definitions of this paper. The second simplest example of this kind is the following:

(b) $H$ generated by $x, y$ and $g^{ \pm 1}$ with relations $x^{2}=0=y^{2}, x y=-y x, g x=-x g$, $g y=-y g$ and comultiplication given by

$$
\begin{gathered}
\Delta g=g \otimes g \\
\Delta x=x \otimes g+1 \otimes x \\
\Delta y=y \otimes g^{-1}+1 \otimes y
\end{gathered}
$$

If we write an element $h \in H$ as

$$
h=\sum_{n \in \mathbb{Z}} a_{n} g^{n}+\sum_{n \in \mathbb{Z}} b_{n} g^{n} x+\sum_{n \in \mathbb{Z}} c_{n} g^{n} y+\sum_{n \in \mathbb{Z}} d_{n} g^{n} x y
$$

then a left integral is given by $\Lambda(h)=d_{0}$. We will compute some invariants of the (stable) comodule category associated to this $H \cong k[\mathbb{Z}] \# \Lambda(x, y)$.

One of the main goals of this paper is to translate into Hopf-co-Frobenius language the notion of homology "Ker $d / \operatorname{Im} d$ ". The definition is very natural:

\subsection{Hopf homology for algebras with a non-zero integral}

Definition 2.1. Given a co-Frobenius Hopf algebra $H$ and $M \in \mathcal{M}^{H}$, denote

$$
\mathcal{H}_{0}^{H}(M):=\frac{M^{c o H}}{\Lambda \cdot M}
$$

For $n \in \mathbb{N}$, we define

$$
\mathcal{H}_{-n}^{H}(M):=\mathcal{H}_{0}^{H}\left(T^{n} M\right)
$$

and

$$
\mathcal{H}_{n}^{H}(M):=\mathcal{H}_{0}^{H}\left(T^{\prime n} M\right)
$$

Example 2.2. If $M=k$ and $H$ is co-Frobenius with $\Lambda(1)=0$, then $\Lambda \cdot k=0$, hence $\mathcal{H}_{0}^{H}(k)=k$ and the functor $\mathcal{H}_{0}^{H}$ is non trivial.

Example 2.3. For $M=H, \Lambda \cdot H=k 1_{H}=H^{c o H} \Rightarrow \mathcal{H}_{0}^{H}(H)=0$.

Example 2.4. The condition " $M^{c o H} / \Lambda \cdot M=0$ " is stable under arbitrary direct sums and direct summands, so $I^{\mathrm{coH}} / \Lambda \cdot I=0$ for any injective module $I$.

As a corollary, if $f: M \rightarrow N$ is an $H$-colinear map such that if factors through an injective:

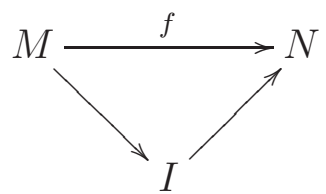


then the induced map

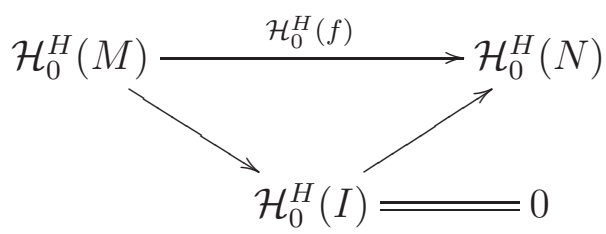

is necessarily zero. So, the functor $\mathcal{H}_{0}^{H}(-)$ is actually defined in the stable category

$$
\mathcal{H}_{0}^{H}: \underline{\mathcal{M}}^{H} \rightarrow{ }_{k} V e c t
$$

Remark 2.5. For all $n \in \mathbb{Z}$, the functors $\mathcal{H}_{n}^{H}(-)$ are defined in the stable category.

Corollary 2.6. If $H$ is co-semisimple then every $H$-comodule is injective, hence $\mathcal{H}_{0}^{H}(M)=0$ for all comodule $M$. In other words, $\Lambda \cdot M=M^{c o H}$ for all comodule $M$.

Remark 2.7. From the point of view of invariant theory, $\Lambda \cdot M=M^{c o H}$ is the most convenient situation, but from the point of view of homological algebra, $\mathcal{H}_{0}^{H}(M) \neq 0$ is most interesting.

Lemma 2.8. Let $H$ be a Hopf algebra with nonzero integral $\Lambda$ and denote $\underline{\operatorname{Hom}}^{H}$ the Hom space in the stable category of $H$ comodules, then there exists an epimorphism

$$
\underline{\operatorname{Hom}}^{H}(k, M) \rightarrow \mathcal{H}_{0}^{H}(M)
$$

Proof. Notice that

$$
\begin{gathered}
\operatorname{Hom}^{H}(k, M) \rightarrow M^{c o H} \\
f \mapsto f(1)
\end{gathered}
$$

is an isomorphism. We will show that this map fits into a commutative square

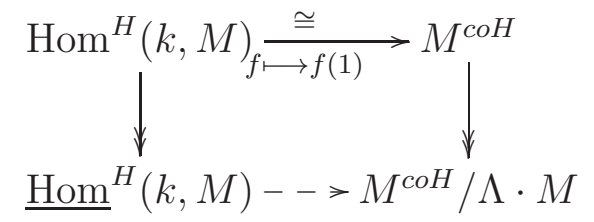

Assume $f: k \rightarrow M$ factors through an injective object

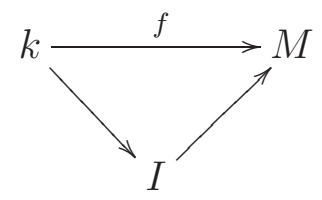

then one may consider the unit map $k \stackrel{\eta}{\rightarrow} H$ and the diagram

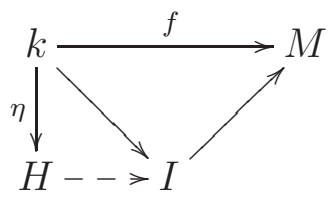


Since $\eta$ is a monomorphism and $I$ is injective, one may find a dashed morphism making a commutative diagram, so, it is enough to consider the case $I=H$.

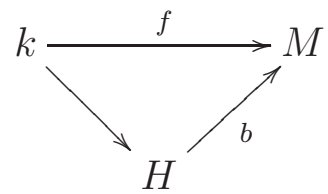

Now if $x \in H$ is such that $\Lambda(x)=1$, then

$$
f(1)=b(1)=b(\Lambda(x) 1)=b(\Lambda \cdot x)=\Lambda \cdot b(x) \in \Lambda \cdot M
$$

so $f(1) \in \Lambda \cdot M$. This proves that the induced map

$$
\underline{\operatorname{Hom}}^{H}(k, M) \rightarrow M^{c o H} / \Lambda \cdot M
$$

is both well-defined, and clearly surjective.

Remark 2.9. One may wonder if the epimorphism of the above Lemma is in fact an isomorphism. This will be the case (see Theorem 2.12). For finite dimensional Hopf algebras it is due to You Qi [Qi2], where he proves actually for finite dimensional Frobenius algebras, in particular for finite dimensional Hopf algebras. It is not clear for the author how to adapt Qi's arguments to our case, maybe one can find a simpler proof, but we provide a proof with some homological machinery first.

Remark 2.10. Lemmas 1.9 and 1.10 gives an alternative proof that the composition of two consecutive morphisms in a triangle is zero (in the stable category), and so, every functor defined in the stable category sends triangles to complexes. For the particular case of $\mathcal{H}_{\bullet}^{H}(-)$, without knowing that it is representable or not, we have the expected result:

Theorem 2.11. If $X \rightarrow Y \rightarrow Z \rightarrow T X$ is a triangle in the stable category then there is a long exact sequence of vector spaces

$$
\cdots \rightarrow \mathcal{H}_{n+1}^{H}(Z) \rightarrow \mathcal{H}_{n}^{H}(X) \rightarrow \mathcal{H}_{n}^{H}(Y) \rightarrow \mathcal{H}_{n}^{H}(Z) \rightarrow \mathcal{H}_{n-1}^{H}(X) \rightarrow \cdots
$$

Proof. We will prove that

$$
\mathcal{H}_{0}^{H}(X) \rightarrow \mathcal{H}_{0}^{H}(Y) \rightarrow \mathcal{H}_{0}^{H}(Z)
$$

is exact in $\mathcal{H}_{0}^{H}(Y)$ when $0 \rightarrow X \rightarrow Y \rightarrow Z \rightarrow 0$ is a short exact sequence. The general result follows from Lemma 1.10 and the shifting axiom of triangles. So assume $0 \rightarrow X \rightarrow Y \rightarrow Z \rightarrow 0$ is a short exact sequence in $\mathcal{M}^{H}$, then multiplication by the integral gives as a commutative diagram (of vector spaces) with exact rows

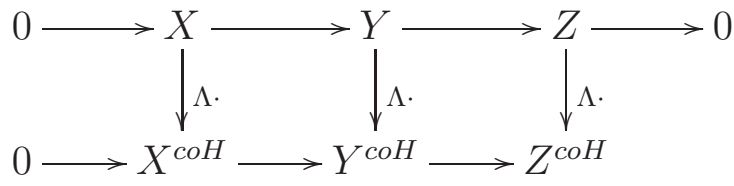

So, even forgetting that $X \rightarrow Y$ is injective, the snake Lemma gives in particular that

$$
X^{\mathrm{coH}} / \Lambda \cdot X \longrightarrow Y^{\mathrm{coH}} / \Lambda \cdot Y \longrightarrow Z^{\mathrm{coH}} / \Lambda \cdot Z
$$

is exact. 
Now, the above Theorem together with Lemma 2.8 gives the following:

Theorem 2.12. Let $H$ be a Hopf algebra with nonzero integral $\Lambda$ and denote $\underline{\mathrm{Hom}}^{H}$ the Hom space in the stable category of $H$ comodules, then the natural map

$$
\underline{\operatorname{Hom}}^{H}(k, M) \rightarrow \mathcal{H}_{0}^{H}(M)
$$

is an isomorphism.

Proof. First recall the following version of the "5"-lemma: given a commutative diagram with exact rows:

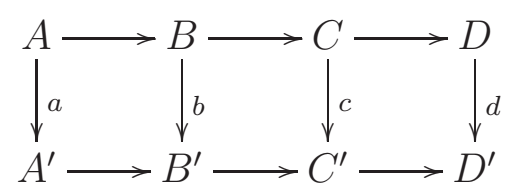

if $b$ and $d$ are monomorphisms and $a$ is an epimorphism, then $c$ is a monomorphism.

Consider $\mathcal{S}$ the class of $H$-comodules $S$ such that the map $\underline{\operatorname{Hom}}^{H}(k, S) \rightarrow \mathcal{H}_{0}^{H}(S)$ is an isomorphism. Because short exact sequences in $\mathcal{M}^{H}$ gives both long exact sequences for $\mathcal{H}_{n}^{H}(-)$ and $\underline{\operatorname{Hom}}^{H}\left(k, T^{-n}(-)\right)$, given a short exact sequence of comodules

$$
0 \rightarrow S_{1} \rightarrow M \rightarrow S_{2} \rightarrow 0
$$

where $S_{1}$ nd $S_{2}$ are in $\mathcal{S}$, then we have a diagram

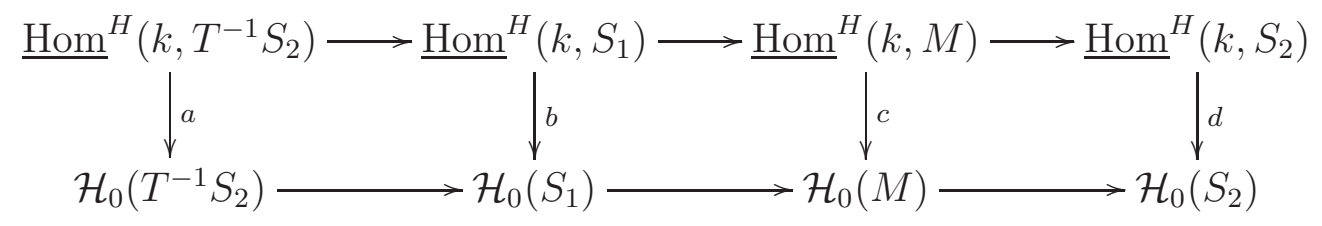

Every vertical map is an epimorphism (Lemma 2.8) and both $b$ and $d$ are monomorphism because they are isomorphisms $\left(S_{i} \in \mathcal{S}\right)$, so $c$ is monomorphism, hence, an isomorphism.

We conclude that the theorem is true for any finite dimensional comodule $M$, provided it is true on simple comodules.

If $S=k$ and $k$ is not injective then $\Lambda \cdot k=0$ and $\mathcal{H}_{0}^{H}(k)=k \cong \underline{\operatorname{Hom}}^{H}(k, k)$. (If $k$ is injective, the theorem is noninteresting, but still true).

If $S$ is simple and $S \neq k$ then $S^{c o H}=0$, so trivially $\mathcal{H}_{0}^{H}(S)=0=\underline{\operatorname{Hom}}(k, S)$.

Now let $M$ be a possibly infinite dimensional comodule and $f: k \rightarrow M$ such that $f(1)=\Lambda \cdot m$ for some $m \ni M$. Consider $M^{\prime} \subset M$ a finite dimensional subcomodule containing $f(1)$ and $m$. Then, the class of $f(1)$ in $\mathcal{H}_{0}^{H}\left(M^{\prime}\right)$ is zero. But because $M^{\prime}$ is finite dimensional we know $\mathcal{H}_{0}^{H}\left(M^{\prime}\right)=\underline{\operatorname{Hom}}^{H}\left(k, M^{\prime}\right)$ and so there exists a factorization

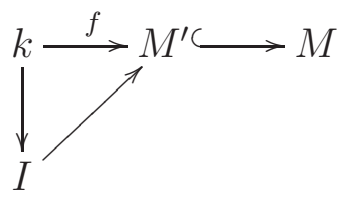

with $I$ injective. So, $f$ is zero in $\underline{\operatorname{Hom}}^{H}(k, M)$. 


\subsection{Multiplicative structure}

Because $H$ is Hopf, the categories $\mathcal{M}^{H}$ and $\mathfrak{m}^{H}$ are tensor categories, and the tensor structure descends to the stable category, as one can see after these standard facts:

Lemma 2.13. 1. If $C$ is a coalgebra and $V$ a vector space, the right $C$ comodule $V \otimes C$ with structure map $\rho=\mathrm{id}_{V} \otimes \Delta$ is an injective comodule.

2. Every injective comodule is a direct summand of one as above. The category of comodules has enough injectives.

Proof. 1. It follows from the adjunction formula

$$
\begin{gathered}
\operatorname{Hom}^{C}(M, V \otimes C) \cong \operatorname{Hom}_{k}(M, V) \\
f \mapsto\left(\operatorname{id}_{V} \otimes \epsilon\right) \circ f
\end{gathered}
$$

and that every vector space is an injective object in $k$-Vect.

2. If $M$ is a comodule, the structure morphism

$$
\rho_{M}: M \rightarrow M \otimes C
$$

gives an embedding into an injective object: $C$-colinearity is by coassociativity and injectivity is because of counitarity. If $M=I$ is injective, then the monomorphism : $\rho_{I}: I \rightarrow I \otimes C$ splits, hence, $I$ is a direct summand of $V \otimes C$ where $V$ is the underlying vector space of $I$.

Lemma 2.14. Let $H$ be a Hopf algebra, $M \in \mathcal{M}^{H}$. Denote $V_{M}$ the underlying vector space of $M$.

1. $M \otimes H$ (with diagonal coaction) is isomorphic to $V_{M} \otimes H$ (with $\rho=\mathrm{id}_{V_{M}} \otimes \Delta_{H}$ ).

2. Also $H \otimes M \cong V_{M} \otimes H$

3. If $I$ is injective then $M \otimes I$ and $I \otimes M$ are both injectives.

Proof. 1. We only exhibit the maps:

$$
\begin{aligned}
& M \otimes H \rightarrow V_{M} \otimes H \\
& m \otimes h \mapsto m_{0} \otimes m_{1} h
\end{aligned}
$$

with inverse

$$
m \otimes h \mapsto m_{0} \otimes S\left(m_{1}\right) h
$$

The composition is

$$
m \otimes h \mapsto m_{0} \otimes S\left(m_{1}\right) m_{2} h=m_{0} \otimes \epsilon\left(m_{1}\right) h=m \otimes h
$$

The other composition is similar. The surprising part is that these maps are $H$ colinear. For instance:

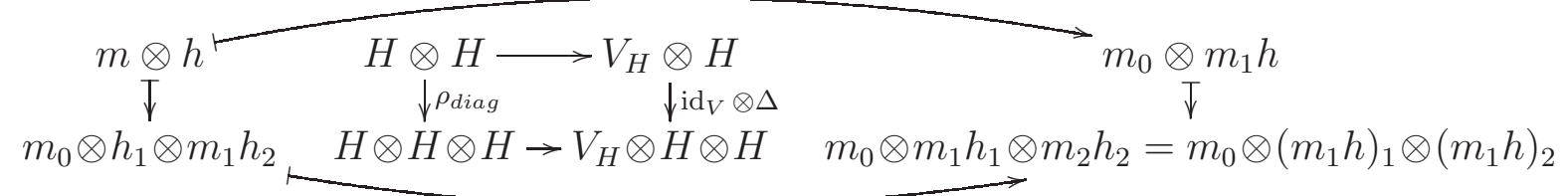


2. The maps are similar: consider

$$
\begin{gathered}
H \otimes M \rightarrow V_{M} \otimes H \\
h \otimes m \mapsto m_{0} \otimes m_{1} h
\end{gathered}
$$

with inverse

$$
m \otimes h \mapsto S\left(m_{1}\right) h \otimes m_{0}
$$

The composition is

$$
h \otimes m \mapsto m_{0} \otimes m_{1} h=S\left(m_{1}\right) m_{2} h \otimes m_{0}=\epsilon\left(m_{1}\right) h \otimes m_{0}=h \otimes m
$$

The other composition is similar. The colinearity follows the same lines.

3. If $I$ is injective then it is isomorphic to a direct summand of $V \otimes H$ for some vector space $V$ (e.g. $\left.V=V_{I}\right)$, and so $M \otimes I$ is isomorphic to a direct summand of

$$
M \otimes(V \otimes H) \cong V \otimes(M \otimes H) \cong\left(V \otimes V_{M}\right) \otimes H
$$

and $I \otimes M$ is a direct summand of

$$
(V \otimes H) \otimes M \cong V \otimes(H \otimes M) \cong\left(V \otimes V_{M}\right) \otimes H
$$

In any case, a direct summand of a comodule of the form $W \otimes H$ for some vector space $W$.

There are several corollaries:

Corollary 2.15. The tensor product is well defined in the stable category. In particular, $K_{0}\left(\underline{\mathfrak{m}}^{H}\right)$ is an associative ring.

Let $E:=E(k)$ be the injective hull of $k$ in $\mathcal{M}^{H}$. It is well-known that $H$ is coFrobenius if and only if $E(k)$ is finite dimensional (see Theorem 2.1 in [AC]). Also, for co-Frobenius Hopf algebras, there exists a finite dimensional projective comodule $P=P(k)$ with a surjective map $P \rightarrow k$.

Corollary 2.16. Define $E(M):=M \otimes E$. The map $M \rightarrow E(M)(m \mapsto m \otimes 1)$ is a functorial injective embedding, if $M \in \mathfrak{m}^{H}$ then $E(M) \in \mathfrak{m}^{H}$ as well. Also, $P(M):=M \otimes P$ gives a functorial projective surjection $P(M) \rightarrow M$, if $P \in \mathfrak{m}^{H}$ then $P(M) \in \mathfrak{m}^{H}$ as well.

Proof. The injective part is clear. Let us prove the existence of a surjective map $P \rightarrow k$ with $P$ finite dimensional:

Since $\Lambda^{\prime}: H \rightarrow k$ is surjective, there exists $h_{0} \in H$ such that $\Lambda^{\prime}\left(h_{0}\right)=1$, and there exists a finite dimensional subcomodule $M_{0} \subset H$ containing $h_{0}$. In particular, $\Lambda^{\prime}\left(M_{0}\right) \neq 0$. Because $H$ is co-Frobenius, there exists a finite dimensional injective hull of $M_{0}$, let's call it $I\left(M_{0}\right)$. Looking at the diagram

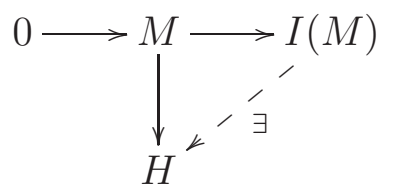


Because $H$ is injective there exist the dashed arrow. Because $I\left(M_{0}\right)$ is the injective hull and $H$ is injective, the map $I(M) \rightarrow H$ is injective and $I\left(M_{0}\right)$ is a direct summand of $H$. Eventually changing $M_{0}$ by $I\left(M_{0}\right)$ we get a finite dimensional direct summand of $H$ such that the restriction of $\Lambda^{\prime}$ is non-zero, hence, a surjection $P \rightarrow k$ with $P$ projective and finite dimensional.

Corollary 2.17. For any $M, N$ in $\mathcal{M}^{H}$, there are isomorphisms in the stable category

$$
T(M \otimes N) \cong T M \otimes T N \cong T M \otimes N \cong M \otimes T N
$$

and similarly for $T^{\prime}$. Hence, $\underline{\mathcal{M}}^{H}$ and $\underline{\mathfrak{m}}^{H}$ are tensor triangulated categories.

Proof. Let $i: M \rightarrow I(M)$ and $j: N \rightarrow I(N)$ be embeddings into injective comodules, then $I(M) \otimes I(N)$ is injective and one can compute $T(M \otimes N)$ via

$$
0 \rightarrow M \otimes N \stackrel{i \otimes j}{\rightarrow} I(M) \otimes I(N) \rightarrow T(M \otimes N) \rightarrow 0
$$

But $I(M) \otimes N$ and $M \otimes I(M)$ are injectives too, and we have the following short exact sequences with injective objects in the middle:

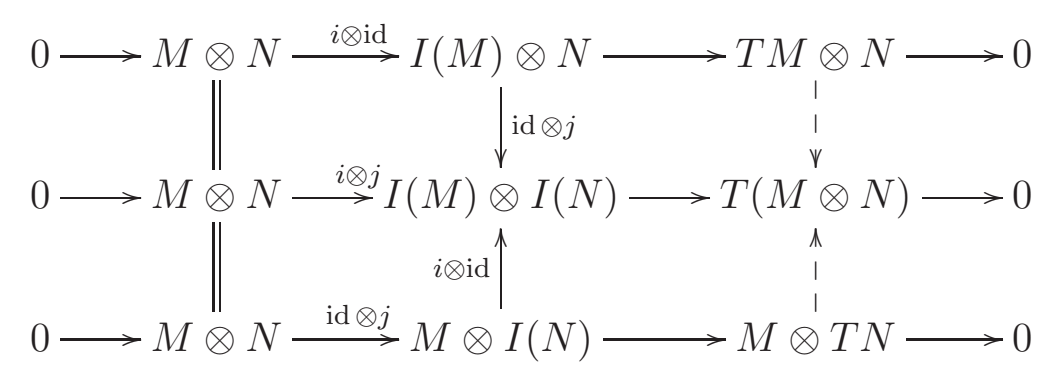

Notice that the morphisms are not canonical in the category of comodules, but they are canonically determined in the stable category

Corollary 2.18. For any $m, n \in \mathbb{Z}$ there is an isomorphism in the stable category

$$
T^{n} M \otimes T^{n} N \cong T^{n+m}(M \otimes N)
$$

\section{Künneth map}

Let $M$ and $N$ be two comodules. It is clear that $M^{H} \otimes N^{H} \subseteq(M \otimes N)^{H}$ and also one can easily check that

$$
\Lambda \cdot\left(M \otimes N^{c o H}\right)=\Lambda \cdot M \otimes N^{c o H}
$$

and

$$
\Lambda \cdot\left(M^{c o H} \otimes N\right)=M^{c o H} \otimes \Lambda \cdot N
$$

So, there is a canonical map

$$
\mathcal{H}_{0}^{H} M \otimes \mathcal{H}_{0}^{H} N=\frac{M^{c o H}}{\Lambda \cdot M} \otimes \frac{N^{c o H}}{\Lambda \cdot N} \cong \frac{M^{c o H} \otimes N^{c o H}}{\Lambda \cdot M \otimes N^{c o H}+M^{c o H} \otimes \Lambda \cdot N} \longrightarrow \mathcal{H}_{0}^{H}(M \otimes N)
$$


Moreover, using Corollary 2.18 on can define maps

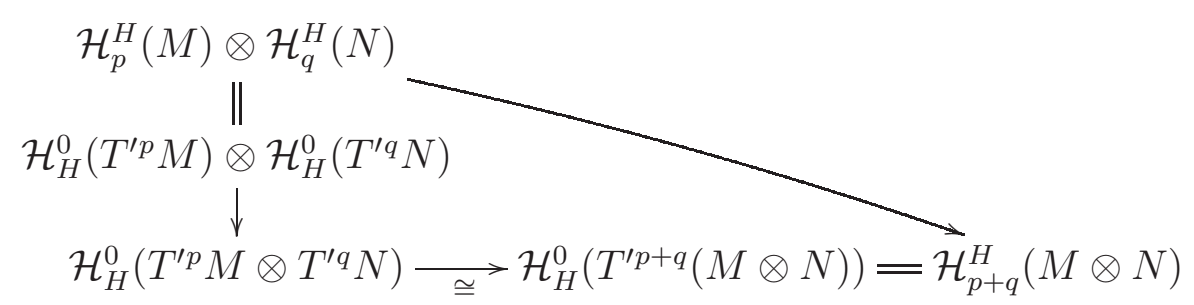

(If a number is negative, we use the convention $\left(T^{\prime}\right)^{-n}=T^{n}$.) In this way, one can assembly all those maps and get, for any fixed $n$, a map that we call "Künneth map"

$$
\bigoplus_{p+q=n} \mathcal{H}_{p}^{H}(M) \otimes \mathcal{H}_{q}^{H}(N) \rightarrow \mathcal{H}_{n}^{H}(M \otimes N)
$$

For $M=N=k$, from concrete computations (see Corollary 4.10) we know this map cannot be an isomorphism in general. It would be interesting to know their general properties. In any case, $\mathcal{H}_{\bullet}^{H}(k)=\bigoplus_{n \in \mathbb{Z}} \mathcal{H}_{n}^{H}(k)$ is a graded algebra.

\section{Small injective embeddings for $\mathfrak{m}^{H_{0} \# \mathfrak{B}}$}

During this section we assume

1. $H_{0}$ is a co-semisimple Hopf algebra,

2. $V \in{ }_{H_{0}} \mathcal{Y} \mathcal{D}^{H_{0}}$ is such that $\mathfrak{B}(V)$, the Nichols algebra associated to the braided vector space $V$, is finite dimensional.

Let us recall briefly the conditions above and set notations and conventions. First, $H_{0} \mathcal{Y} \mathcal{D}^{H_{0}}$ is the category whose objects are left $H_{0}$-modules and right $H_{0}$-comodules with the compatibility

$$
\left.h_{1} m_{0} \otimes h_{2} m_{1}=\left(h_{2} m\right)_{0} \otimes\left(h_{2} m\right)_{1}\right) h_{1}
$$

where $h_{1} \otimes h_{2}=\Delta h, h \in H_{0}$ and $m \in M, \rho(m)=m_{0} \otimes m_{1} \in M \otimes H_{0}$. Morphisms are $H_{0}$-linear and colinear maps. For any Hopf algebra $A$, the category ${ }_{A} \mathcal{Y} \mathcal{D}^{A}$ is braided with

$$
\begin{gathered}
c_{V, W}: V \otimes W \rightarrow W \otimes V \\
v \otimes w \mapsto w_{0} \otimes w_{1} \cdot v
\end{gathered}
$$

Recall that if $V$ is a braided vector space (e.g. $V \in \mathcal{Y} \mathcal{D}_{H_{0}}^{H_{0}}$ ) then both $T V$ (the tensor algebra) and $T^{c} V$ (the tensor coalgebra) are braided Hopf algebras. $T V$ has free product and braided-shuffle coproduct, while $T^{c} V$ has deconcatenation coproduct and braided-shuffle product. The Nichols algebra $\mathfrak{B}(V)$ is, by definition, the image of the unique (bi)algebra map $T V \rightarrow T^{c} V$ that is the identity on $V$ :

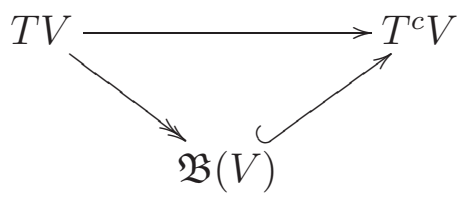


It happens to be, degree by degree, the image of the quantum symmetrizer map associated to the braiding. We refer to Andruskievitch's notes [A] for a gentle introduction and full discussion on Nichols algebras. The reader may keep in mind the easy example $\mathfrak{B}(V)=\Lambda V$ when the braiding is -flip. The braided bialgebra $\mathfrak{B}(V)$ is actually a braided Hopf algebra, and the bicross product $H_{0} \# \mathfrak{B}(V)$ is a usual Hopf algebra. Since there is a lot of structures around $\mathfrak{B}(V)$ we recall them:

- $\mathfrak{B}$ is a coalgebra, we denote $\Delta(b)=b_{1} \otimes b_{2}$,

- $\mathfrak{B} \in \mathcal{M}^{H_{0}}$, we denote the structure $\rho(b)=b_{0} \otimes b_{1}$,

- $H_{0} \# \mathfrak{B}$ is a coalgebra, the comultiplication is given by the following diagram (recall the underlying vector space of $H_{0} \# \mathfrak{B}$ is $H_{0} \otimes \mathfrak{B}$ ):

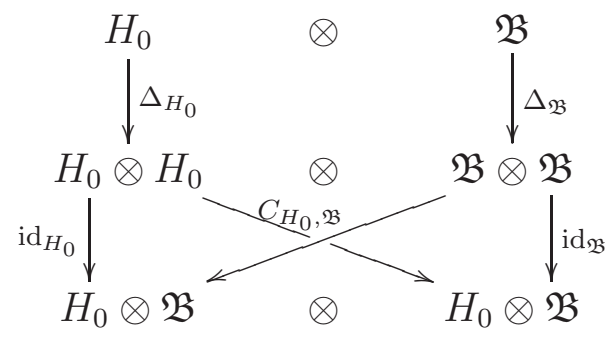

In Sweedler-type notation:

$$
\Delta(h \# b)=h_{1} \#\left(b_{1}\right)_{0} \otimes\left(b_{1}\right)_{1} h_{2} \# b_{2}
$$

- In particular $\Delta(1 \# b)=1 \#\left(b_{1}\right)_{0} \otimes\left(b_{1}\right)_{1} \# b_{2}$. Denoting $H:=H_{0} \# \mathfrak{B}$, we have that $\mathfrak{B} \cong 1 \# \mathfrak{B}$ is a right $H$-subcomodule of $H$. With this structure we consider $\mathfrak{B}$ as an object in $\mathcal{M}^{H}$. To emphasize the difference with $\rho: B \rightarrow \mathfrak{B} \otimes H_{0}$ we call it $\rho_{H}$.

Example 3.1. Let $x \in V \subset \mathfrak{B}, \Delta x=x \otimes 1+1 \otimes x$. Assume $\rho(x)=x \otimes g \in \mathfrak{B} \otimes H_{0}$. In order to compute $\rho_{H}(x)$ we proceed as follows:

$$
\begin{gathered}
\rho_{H}(x) \leftrightarrow \Delta_{H}(1 \# x)=1 \#\left(x_{1}\right)_{0} \otimes\left(x_{1}\right)_{1} \# x_{2} \\
=1 \#(x)_{0} \otimes(x)_{1} \# 1+1 \#(1)_{0} \otimes(1)_{1} \# x \\
=1 \# x \otimes g \# 1+1 \# 1 \otimes 1 \# x \leftrightarrow x \otimes g+1 \otimes x
\end{gathered}
$$

The main fact of this section is the following:

Proposition 3.2. $\mathfrak{B} \in \mathcal{M}^{H}$ is an injective object.

Proof. Since $H_{0}$ is cosemisimple, the inclusion $k \rightarrow H_{0}$ splits as $H_{0}$-comodule. Choose a splitting $\Lambda_{0}^{\prime}: H_{0} \rightarrow k$. This is actually right integral for $H_{0}$, that is, it satisfies

$$
\Lambda_{0}^{\prime}\left(h_{1}\right) h_{2}=\Lambda_{0}^{\prime}(h) 1
$$

and additionally $\Lambda_{0}^{\prime}(1)=1$.

Now we define a splitting $H \rightarrow \mathfrak{B}$ of the inclusion $\mathfrak{B} \cong 1 \# \mathfrak{B} \subset H_{0} \# \mathfrak{B}=H$ via

$$
h \# b \mapsto \Lambda_{0}^{\prime}(h) b
$$


We need to see that it is $H$-colinear. Recall the $H$-structure in $\mathfrak{B}$ is given by the identification $\mathfrak{B} \cong 1 \# \mathfrak{B} \subset H$, so

$$
\rho_{H}(b)=b_{1_{0}} \otimes b_{1_{1}} \# b_{2}
$$

We check $H$-colinearity:

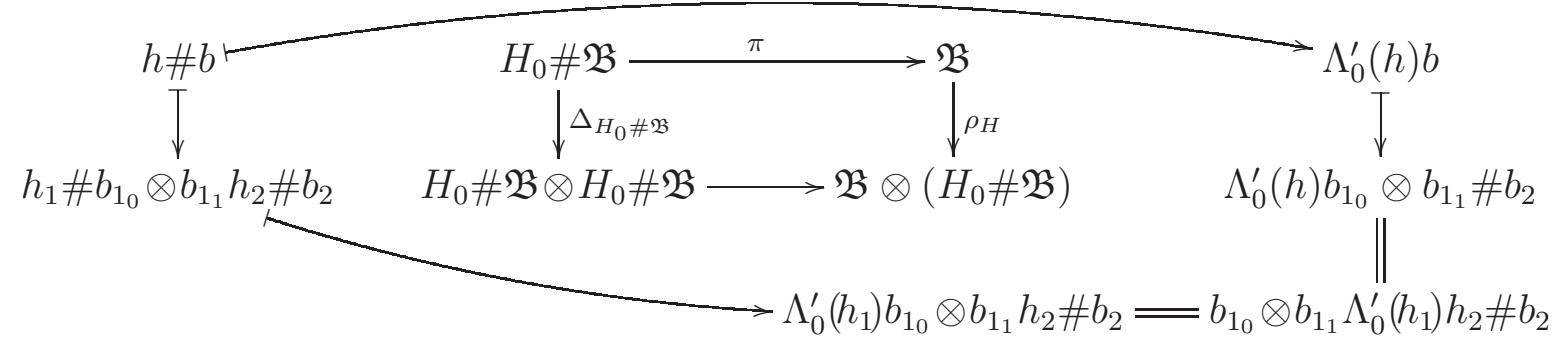

Remark 3.3. The proof is independent of the fact of $\mathfrak{B}$ being finite dimensional, but we are interested in the case $\operatorname{dim} \mathfrak{B}<\infty$ so that $H_{0} \# \mathfrak{B}$ is co-Frobenius.

As a corollary we have

Corollary 3.4. For any $M \in \mathcal{M}^{H}$, the map $i_{M}: M \rightarrow M \otimes \mathfrak{B}$ defined by

$$
m \mapsto m \otimes 1
$$

is a functorial injective embedding. In particular, if $\mathfrak{B}$ is finite dimensional then $M \rightarrow M \otimes \mathfrak{B}$ $(m \mapsto m \otimes 1)$ is a finite dimensional embedding working in $\mathfrak{m}^{H}$. From the short exact sequence

$$
0 \rightarrow M \rightarrow M \otimes \mathfrak{B} \rightarrow(M \otimes \mathfrak{B}) / M \rightarrow 0
$$

we have $T M \cong(M \otimes \mathfrak{B}) / M$. Recall $\mathfrak{B}$ is graded (with the tensor grading) and $\mathfrak{B}_{\text {top }}$ (its maximal degree) has dimension 1. The Kernel of $\left.\Lambda^{\prime}\right|_{\mathfrak{B}}$ is $\mathfrak{B}_{<t o p}=\oplus_{i=0}^{\text {top-1 }} \mathfrak{B}_{i}$. From $\pi: \mathfrak{B} \rightarrow$ $\mathfrak{B} / \mathfrak{B}_{<\text {top }}$ we have the short exact sequence

$$
0 \rightarrow M \otimes \mathfrak{B}_{<\text {top }} \rightarrow M \otimes \mathfrak{B} \rightarrow M \otimes\left(\mathfrak{B} / \mathfrak{B}_{<\text {top }}\right) \rightarrow 0
$$

hence $T^{\prime}\left(M \otimes\left(\mathfrak{B} / \mathfrak{B}_{<\text {top }}\right)\right) \cong M \otimes \mathfrak{B}_{<\text {top }}$.

Remark 3.5. $\mathfrak{B} / \mathfrak{B}_{<t o p}$ is not isomorphic to $k$ in general, but it is 1 -dimensional. So, in order to compute $T^{\prime} M$ one should "twist $M \otimes \mathfrak{B}_{<\text {top }}$ by the inverse of the quantum determinant":

If $\mathfrak{b}$ is a generator of the 1 -dimensional vector space $\mathfrak{B}_{\text {top }}$ then $k \mathfrak{b}$ is not in general an $H$-subcomodule of $\mathfrak{B}$, but $\mathfrak{B} / \mathfrak{B}_{<\text {top }}=k \overline{\mathfrak{b}}$ is so, hence

$$
\rho_{H}(\overline{\mathfrak{b}})=\overline{\mathfrak{b}} \otimes D
$$

for a unique group-like element $D \in H$, that we call "quantum determinant". From the surjective map

$$
\mathfrak{B} \stackrel{\pi}{\rightarrow} \mathfrak{B} / \mathfrak{B}_{<\text {top }} \cong k D
$$

we get a surjective map into the trivial comodule $k$ :

$$
\mathfrak{B} \otimes k D^{-1} \rightarrow k
$$


If we call $P:=\mathfrak{B} \otimes k D^{-1}$, it is a projective $H$-comodule that surjects into $k$ and from it one has functorial projective surjections for any comodule $M$ :

$$
P(M):=M \otimes P \rightarrow M
$$

and functorial $T^{\prime}$, since from:

$$
0 \rightarrow M \otimes \mathfrak{B}_{<\text {top }} \otimes k D^{-1} \rightarrow M \otimes P \rightarrow M \rightarrow 0
$$

we get $T^{\prime}(M):=M \otimes B_{<t o p} \otimes k D^{-1}$ is a functor in $\mathcal{M}^{H}$ (resp. in $\mathfrak{m}^{H}$ if $M$ is finite dimensional) that gives the desuspension functor in $\underline{\mathcal{M}}^{H}\left(\right.$ resp. in $\left.\underline{\mathfrak{m}}^{H}\right)$.

Before going into $K_{0}$ rings, we look at some examples.

\section{First examples}

\subsection{The example $k[\mathbb{Z}] \# k[x] / x^{2}$}

Let $H$ be the $k$-algebra generated by $x$ and $g^{ \pm 1}$ with relations

$$
g x=-x g, x^{2}=0
$$

It is a Hopf algebra if one defines the comultiplication by

$$
\Delta g=g \otimes g, \Delta x=x \otimes g+1 \otimes x
$$

(to be compared with Example 3.1). That is, $g$ is group-like and $x$ is 1- $g$-primitive. Notice that $k[x] / x^{2}$ is not a Hopf algebra in the usual sense (unless characteristic=2), but it is a super Hopf algebra. Nevertheless, $H=k[\mathbb{Z}] \#\left(k[x] / x^{2}\right)$ is a Hopf algebra in the usual sense. Maybe all computations in this example are folklore, but for clearness we include them.

For an element

$$
\omega=\sum_{n \in \mathbb{Z}} a_{n} g^{n}+\sum_{n \in \mathbb{Z}} b_{n} g^{n} x
$$

define

$$
\Lambda(\omega):=b_{0}
$$

The main fact about the category $\mathcal{M}^{H}$, noticed by Bodo Pareigis $[\mathrm{P}]$, is

$$
\text { A right } H \text {-comodule } M \text { is the same as a d.g. structure on } M
$$

Notice that evaluation at $x=0$ gives a map $H \rightarrow k[\mathbb{Z}]$, so any $H$-comodule is a $k[\mathbb{Z}]$-comodule (i.e. a $\mathbb{Z}$-graded object), but the presence of $x$ keep track of a square-zero differential. We just write the correspondence: if $M=\left(\oplus_{n \in \mathbb{Z}} M_{n}, \partial\right)$ with $\partial\left(M_{n}\right) \subseteq M_{n-1}$ and $\partial^{2}=0$ then, for $m \in M_{n}$, the right comodule structure is

$$
\rho(m)=m \otimes g^{n}+\partial(m) \otimes x g^{n-1}
$$


and every right $H$-comodule is of this form.

It is a pleasant exercise to check that the standard differential on the tensor products of complexes with the usual Koszul $\partial \otimes 1 \pm 1 \otimes \partial$ agree with the standard $H$-comodule structure on the tensor product of $H$-comodules.

Notice that

$$
\begin{gathered}
\Delta 1=1 \otimes 1 \\
\Delta x=x \otimes g+1 \otimes x
\end{gathered}
$$

means that $k[x] / x^{2}$ is a right $H$-subcomodule of $H$. As d.g. vector space is the complex

$$
\cdots \rightarrow 0 \rightarrow k x \underset{x \mapsto 1}{\stackrel{\partial}{\rightarrow}} k \rightarrow 0 \rightarrow \cdots
$$

where $|x|=1,|1|=0$.

Smaller injective embeddings for $H=k[\mathbb{Z}] \# k[x] / x^{2}$

In this case we have $\mathfrak{B}=k \oplus k x$, considered as $H$-comodule via

$$
\begin{gathered}
\rho(1)=1 \otimes 1 \\
\rho(x)=x \otimes g+1 \otimes x
\end{gathered}
$$

The general argument developed in the previous section gives us that, for any $M \in$ $\mathcal{M}^{H}$, the map $M \rightarrow I(M):=M \otimes \mathfrak{B}=M \otimes k[x] / x^{2}$ given by $i(m)=m \otimes 1$ is an embedding of $M$ into an injective object. In particular $I(M) / \rho(M)=M \otimes x$ and we have proven the following:

Corollary 4.1. In the stable category of $\mathcal{M}^{H}$ for $H=k[\mathbb{Z}] \#\left(k[x] / x^{2}\right)$,

$$
T M=I(M) / M \cong M \otimes k x \cong M[1]
$$

We leave as an exercise the following:

Corollary 4.2. Identifying d.g.Vect ${ }_{k}$ and $\mathcal{M}^{H}$, the comodule $M \otimes k[x] / x^{2}$ identifies with the mapping cone of the identity of $M$. Moreover, the "stable H-comodule mapping cone" of a colinear map identifies with the classical mapping cone of a map between complexes.

\section{Homology}

For a d.g. vector space $M=\left(\oplus_{n} M_{n}, \partial\right)$ viewed as $k[\mathbb{Z}] \# k[x] / x^{2}$-comodule, the coinvariants are

$$
M^{c o H}=\left\{m: \rho(m)=m \otimes 1_{H}=m \otimes g^{0}\right\}
$$

But " $\rho(m)=m \otimes g^{0} "$ means that $m \in M_{0}$ and $\partial m=0$, so

$$
M^{c o H}=\operatorname{Ker}\left(\partial: M_{0} \rightarrow M_{-1}\right)
$$

On the other side, the action of the integral on an element $m$ gives

$$
\Lambda \cdot m=(\mathrm{id} \otimes \Lambda) \rho(m)=(\mathrm{id} \otimes \Lambda) \rho\left(\sum_{n} m_{n}\right)
$$




$$
=(\mathrm{id} \otimes \Lambda)\left(\sum_{n}\left(m_{n} \otimes g^{n}+\partial\left(m_{n}\right) \otimes g^{n-1} x\right)\right)=\partial\left(m_{1}\right) \in M_{0}
$$

That is

$$
\Lambda \cdot\left(\oplus_{n} M_{n}, \partial\right)=\operatorname{Im}\left(\partial: M_{1} \rightarrow M_{0}\right) \subseteq \operatorname{Ker}\left(\partial: M_{0} \rightarrow M_{-1}\right) \subseteq M_{0}
$$

Hence

$$
\mathcal{H}_{0}^{k[\mathbb{Z}] \# k[x] / x^{2}}(M)=M^{c o H} / \Lambda \cdot M=H_{0}(M, \partial)
$$

\subsection{The example $k[\mathbb{Z}] \# k[x] / x^{N}$ and $N$-complexes}

Fix $N \in \mathbb{N}, N \geq 2$. Let $H$ be the algebra generated by $g^{ \pm 1}$ and $x$ with the relations

$$
x^{N}=0, g x=\xi_{N} x g
$$

where $\xi_{N}$ is an $N$-primitive root of unity. This algebra is Hopf with comultiplication

$$
\Delta g=g \otimes g, \Delta x=x \otimes g+1 \otimes x
$$

To have an $H$-comodule is the same as a $\mathbb{Z}$-graded vector space together with a degree -1 map $\partial$ satisfying $\partial^{N}=0$. The tensor structure for $\left(M_{\bullet}, \partial_{M}\right) \otimes\left(M_{\bullet}^{\prime}, \partial_{R}\right)$ is given by the usual total grading in $M \otimes M^{\prime}$, and the differential on homogeneous elements is

$$
\partial\left(m \otimes m^{\prime}\right)=\partial(m) \otimes m^{\prime}+\xi_{N}^{|m|} m \otimes \partial\left(m^{\prime}\right)
$$

For an homogeneous element $m \in M$ of degree $n$, the coaction is given by

$$
\begin{gathered}
\rho(m)=m \otimes g^{n}+\partial(m) \otimes x g^{n-1}+\frac{1}{[2]_{\xi}} \partial^{2}(m) \otimes x^{2} g^{n-2}+\cdots+\frac{1}{[N-1]_{\xi} !} \partial^{N-1}(m) \otimes x^{N-1} g^{n-N+1} \\
=\sum_{i=1}^{N-1} \frac{1}{[i]_{\xi} !} \partial^{i}(m) \otimes x^{i} g^{n-i}
\end{gathered}
$$

where as usual $[0]_{\xi} !=[1]_{\xi} !=1,[n]_{\xi}=1+\xi+\cdots+\xi^{n-1}$ and $[n+1] !_{\xi}=[n+1]_{\xi} \cdot[n] !_{\xi}$.

If $\left(M_{\bullet}, \partial\right)$ is an $N$-complex, there are several ways to associate an "homology" in degree $n$. For each $0<i<N$, since $0=\partial^{N}=\partial^{i} \partial^{N-i}$, one may consider $\operatorname{Ker}\left(\partial^{i}\right) / \operatorname{Im}\left(\partial^{N-i}\right)$. The general machinery of co-Frobenius algebras and stable categories, however, choose one particular $i$. Since $M^{c o H}=\operatorname{Ker}(\partial) \cap M_{0}$ and $\Lambda \cdot M=M_{0} \cap \operatorname{Im}\left(\partial^{N-1}\right)$ we have

$$
\mathcal{H}_{0}^{H}(M)=\frac{\left\{m \in M_{0}: \partial(m)=0\right\}}{\partial^{N-1}\left(M_{-N+1}\right)}
$$

The other (homological) degrees are not the $\mathcal{H}_{0}$ of the degree-shiftings of the $N$-complex. As an illustration we compute $\mathcal{H}_{1}(M)$ in terms of the $N$-complex data:

Proposition 4.3. $H_{1}(M) \cong \frac{\operatorname{Ker}\left(\partial^{N-1}\right) \cap M_{N-1}}{\operatorname{Im}\left(\partial: M_{N} \rightarrow M_{N-1}\right)}$

Remark 4.4. From Corollary 3.4 and the isomorphism $\mathfrak{B} / \mathfrak{B}_{<\text {top }} \cong k g^{N-1}$ (notice $\mathfrak{B} / \mathfrak{B}_{<\text {top }}$ is generated by the class of $x^{N-1}$ and $\rho_{H}\left(x^{N-1}\right)=x^{N-1} \otimes g^{N-1}+$ lower degree terms) it follows that

$$
T^{\prime}(M) \cong M \otimes k g^{-N+1} \otimes \mathfrak{B}_{<t o p}
$$


Proof. Recall $\mathfrak{B}=\bigoplus_{i=0}^{N-1} k x^{i}$, the structure is given by

$$
\begin{gathered}
\left|x^{i}\right|=i, \partial\left(x^{i}\right)=[i] x^{i-1} \\
\mathcal{H}_{1}(M)=\mathcal{H}_{0}\left(T^{\prime} M\right)=\mathcal{H}_{0}\left(M \otimes k g^{-N+1} \otimes\left(k \oplus k x \oplus k x^{2} \oplus \cdots \oplus k x^{N-2}\right)\right)
\end{gathered}
$$

The degree zero part of $T^{\prime} M$, if $M=\oplus_{n} M_{n}$, is

$$
T^{\prime}(M)_{0}=\bigoplus_{i=0}^{N-2} M_{N-i-1} \otimes g^{-N+1} \otimes x^{i}
$$

A typical element is of the form

$$
\sum_{i=0}^{N-2} m_{i} \otimes g^{N-1} \otimes x^{i}
$$

where $\left|m_{i}\right|=-N-i+1$. The differential has the form

$$
\begin{gathered}
\partial\left(\sum_{i=0}^{N-2} m_{i} \otimes g^{N-1} \otimes x^{i}\right)=\sum_{i=0}^{N-2} \partial\left(m_{i}\right) \otimes g^{N-1} \otimes x^{i}+\sum_{i=0}^{N-2} \xi_{N}^{-N-i+1+N-1} m_{i} \otimes g^{N-1} \otimes \partial\left(x^{i}\right) \\
=\sum_{i=0}^{N-2} \partial\left(m_{i}\right) \otimes g^{N-1} \otimes x^{i}+\sum_{i=1}^{N-2} \xi_{N}^{-i} m_{i} \otimes g^{N-1} \otimes[i] x^{i-1} \\
=\partial\left(m_{N-2}\right) \otimes g^{N-1} \otimes x^{N-2}+\sum_{i=0}^{N-3}\left(\partial\left(m_{i}\right)+\xi_{N}^{-i-1}[i+1] m_{i+1}\right) \otimes g^{N-1} \otimes x^{i}
\end{gathered}
$$

This expression is equal to zero if and only if

$$
\partial\left(m_{N-2}\right)=0 \quad \text { and } \quad m_{i}=\frac{-\xi_{N}^{i}}{[i]} \partial\left(m_{i-1}\right) \quad(i=N-3, N-4, \ldots, 1)
$$

From the second set of equalities we see that the only parameter is $m_{0}$, because $m_{i}$ is, up to scalar, $\partial^{i}\left(m_{0}\right)$. The equation $\partial\left(m_{N-2}\right)=0$ means $\partial^{N-1}\left(m_{0}\right)=0$. We conclude

$$
\left(T^{\prime} M\right)^{c o H} \cong \operatorname{Ker}\left(\partial^{N-1}\right) \cap M_{N-1}
$$

We leave to the reader to check that, under this bijection, $\Lambda \cdot\left(M \otimes g^{N-1} \otimes \mathfrak{B}_{<\text {top }}\right)$ corresponds to $\partial\left(M_{N}\right)$.

\subsection{The example $k[\mathbb{Z}] \# \Lambda(x, y)$ and Mixed complexes}

Denote

$$
\Lambda(x, y):=k\{x, y\} /\left(x^{2}, y^{2}, x y+y x\right)
$$

It is not a Hopf algebra in the usual sense, but it is a Hopf algebra in the (signed) graded sense. The algebra

$$
k[\mathbb{Z}] \# \Lambda(x, y)=k\left\{g^{ \pm 1}, x, y\right\} /\left(g x=-x g, g y=-y g, 0=x^{2}=y^{2}=x y+y x\right)
$$


is a Hopf algebra with comultiplication

$$
\Delta g=g \otimes g, \Delta x=x \otimes g+1 \otimes x, \Delta y=y \otimes g^{-1}+1 \otimes y
$$

Notice that $x$ produce a differential of degree -1 , while $y$ produce a differential of degree +1 . This Hopf algebra $H$ is isomorphic to $H_{0} \# \mathfrak{B}(V)$ where $H_{0}=k[\mathbb{Z}]$ and $V=k x \oplus k y \in{ }_{H_{0}} \mathcal{Y} \mathcal{D}^{H_{0}}$. Writing $\mathbb{Z}$ multiplicatively $\mathbb{Z} \cong\left\{g^{n}: n \in \mathbb{Z}\right\}$, the action is given by

$$
g v=-v, \forall v \in V
$$

and the coaction is determined by

$$
\rho x=x \otimes g, \rho y=y \otimes g^{-1}
$$

Lemma 4.5. $\mathcal{M}^{H}$ identifies with objects $(M, d, B)$ where $M$ is a $\mathbb{Z}$-graded vector space, $d$ and $B$ are square zero differentials with $|d|=-1,|B|=1$, and $d B+B d=0$. In other words, $\mathcal{M}^{H}$ are mixed complexes.

The proof is straightforward, we only indicate the correspondence: for a mixed complex $(M, d, B)$, the corresponding right comodule structure

$$
\rho: M \rightarrow M \otimes H
$$

for an homogeneous $m$, is given by

$$
\rho(m)=m \otimes g^{|m|}+d(m) \otimes x g^{|m|-1}+B(m) \otimes y g^{|m|+1}+d B(m) \otimes y x g^{|m|}
$$

It is clear that $M^{c o H}=M_{0} \cap \operatorname{Ker} d \cap \operatorname{Ker} B$. Also, an easy computation shows (see Example 6(b) of Section 2] for the expression of the integral)

$$
\Lambda \cdot M=d\left(B\left(M_{0}\right)\right)=B\left(d\left(M_{0}\right)\right) \subseteq M^{c o H}
$$

So,

$$
\mathcal{H}^{0}(M)=\frac{M_{0} \cap \operatorname{Ker} d \cap \operatorname{Ker} B}{d\left(B\left(M_{0}\right)\right)}
$$

Remark 4.6. In this (stable) category, the suspension functor is not the shifting degree in general. However, we have the following lemma:

Lemma 4.7. $k[x] / x^{2}$ and $k[y] / y^{2}$ are $H$-subcomodules of $H . \mathfrak{B}=\Lambda(x, y) \cong k[x] / x^{2} \otimes k[y] / y^{2}$ as objects in $\mathcal{M}^{H}$. For $M \in \mathcal{M}^{H}$ denote $M(x):=M \otimes k[x] / x^{2}$ and $M(y):=M \otimes k[y] / y^{2}$. The following assertions follows:

- $M(x)(y) \cong M(y)(x) \cong M \otimes \Lambda(x, y)$ is an injective object in $\mathcal{M}^{H}$.

- $T(M(x)) \cong M(x)[-1]$,

- $T(M(y)) \cong M(y)[1]$

- $\mathcal{H}_{\bullet}(M(x))=H_{-\bullet}(M, B)$

- $\mathcal{H}_{\bullet}(M(y))=H_{\bullet}(M, d)$ 
Proof. The first item follows from the obvious isomorphism

$$
k[x] / x^{2} \otimes k[y] / y^{2} \cong \Lambda(x, y)
$$

Observe that $k x=\left(k[x] / x^{2}\right) / k=k x \cong k[1]$ and $k y=\left(k[y] / y^{2}\right) / k=k y \cong k[-1]$. Now from the short exact sequence

$$
0 \rightarrow M(x) \rightarrow M(x) \otimes k[y] / y^{2} \rightarrow M(x) \otimes y \rightarrow 0
$$

we get

$$
0 \rightarrow M(x) \rightarrow M \otimes \Lambda(x, y) \rightarrow M(x)[-1] \rightarrow 0
$$

Since $M \otimes \Lambda(x, y)$ is injective, we conclude $T(M(x)) \cong M(x)[-1]$. Similarly for $M(y)$.

In order to compute cohomology we consider first

$$
M(x)^{c o H}=M(x)_{0} \cap \operatorname{Ker} d \cap \operatorname{Ker} B=M(x)_{0}^{d, B}
$$

We visualize it using the following diagram

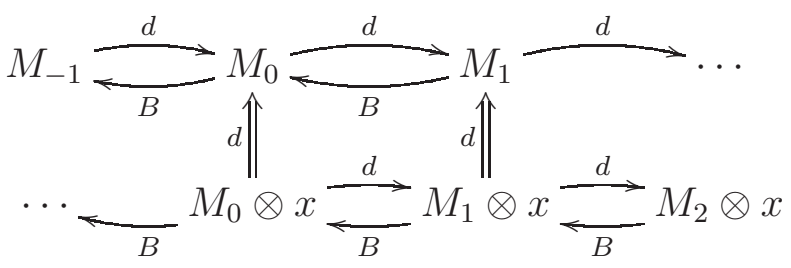

So,

$$
\begin{gathered}
M(x)^{d}=\left\{\left(m_{0}, m_{1} \otimes x\right): d\left(m_{0}\right)+m_{1}=0, d\left(m_{1}\right)=0\right\} \\
=\left\{\left(m_{0},-d m_{0} \otimes x\right): m_{0} \in M_{0}\right\} \cong M_{0} \\
M(x)^{d, B}=\left\{\left(m_{0},-d m_{0} \mid x\right): B m_{0}=0, B\left(-d m_{0}\right)=0\right\}
\end{gathered}
$$

but $B\left(-d m_{0}\right)=d B m_{0}=0$, so $M(x)^{d, B} \cong M_{0}^{B}$.

We also must compute $B\left(d\left(M(x)_{0}\right)\right)$ :

$$
\begin{gathered}
B d\left(m_{0}, m_{1} \otimes x\right)=B\left(d m_{0}+m_{1}, d m_{1} \otimes x\right)=\left(B d m_{0}+B m_{1}, B d m_{1} \otimes x\right) \\
=\left(B\left(m_{1}+d m_{0}\right),-d\left(B\left(m_{1}+d m_{0}\right)\right) \otimes x\right)=(B \widetilde{m},-d(B \widetilde{m}) \otimes x)
\end{gathered}
$$

So, under the isomorphism $M(x)^{d, B} \cong M_{0}^{B}$, the subspace $\Lambda \cdot M(x)=B d\left(M(x)_{0}\right)$ corresponds to $B\left(M_{1}\right) \subset M_{0}$. We conclude $\mathcal{H}^{0}(M(x)) \cong H_{0}(M, B)$.

Now from the second item we get

$$
\mathcal{H}_{n}(M(x))=\mathcal{H}_{0}\left(T^{-n}(M(x))\right)=\mathcal{H}_{0}(M(x)[n])=H_{0}(M(x)[n])=H_{-n}(M, B)
$$

The parts with $M(y)$ instead of $M(x)$ is completely analogous.

Corollary 4.8. For any mixed complex $(M, d, B)$ there are long exact sequences

$$
\cdots \rightarrow \mathcal{H}_{\bullet}(M) \rightarrow H_{\bullet}(M, d) \rightarrow \mathcal{H}_{\bullet}(M[-1]) \rightarrow \mathcal{H}_{\bullet-1}(M) \rightarrow \cdots
$$

and

$$
\cdots \rightarrow \mathcal{H}_{\bullet}(M) \rightarrow H_{-\bullet}(M, B) \rightarrow \mathcal{H}_{\bullet}(M[1]) \rightarrow \mathcal{H}_{\bullet-1}(M) \rightarrow \cdots
$$


Proof. We consider the short exact sequences in $\mathcal{M}^{H}$ :

$$
0 \rightarrow M \rightarrow M \otimes k[y] / y^{2} \rightarrow M \otimes y \rightarrow 0
$$

and

$$
0 \rightarrow M \rightarrow M \otimes k[x] / x^{2} \rightarrow M \otimes x \rightarrow 0
$$

Recall $M \otimes y \cong M[-1]$ and $M \otimes x \cong M[1]$. These short exact sequences in $\mathcal{M}^{H}$ gives triangles in the stable category; their log exact sequences together with the previous Lemma gives the result.

Corollary 4.9. $H_{n}(M, d)=0 \forall n \Rightarrow \mathcal{H}_{n}(M)=\mathcal{H}_{0}(M[-n])$; $H_{n}(M, B)=0 \forall n \Rightarrow \mathcal{H}_{n}(M)=\mathcal{H}_{0}(M[n])$.

Another corollary is the following computation:

Corollary 4.10. Considering $k$ as trivial mixed complex concentrated in degree zero,

$$
\mathcal{H}_{\bullet}(k)= \begin{cases}k & \bullet=0 \\ k & \bullet=-1 \\ 0 & \text { otherwise }\end{cases}
$$

Proof. Specializing the long exact sequence

$$
\cdots \rightarrow \mathcal{H}_{\bullet}(M) \rightarrow H_{\bullet}(M, d) \rightarrow \mathcal{H}_{\bullet}(M[1]) \rightarrow \mathcal{H}_{\bullet-1}(M) \rightarrow H_{\bullet-1}(M, d) \rightarrow \cdots
$$

at $M=k[p]$ and $\bullet=q+1$ gives

$$
\cdots \rightarrow H_{q+1}(k[p], d) \rightarrow \mathcal{H}_{q+1}(k[p+1]) \rightarrow \mathcal{H}_{q}(k[p]) \rightarrow H_{q}(k[p], d) \rightarrow \cdots
$$

If $p \neq q, q+1$ we have

$$
\mathcal{H}_{q+1}(k[p+1]) \cong \mathcal{H}_{q}(k[p])
$$

Inductively, for $n \neq 0,1$

$$
\mathcal{H}_{n}(k)=\mathcal{H}_{n}(k[0]) \cong \mathcal{H}_{n-1}(k[-1]) \cong \cdots \cong \mathcal{H}_{0}(k[-n])=0
$$

because $k[n]$ do not have 0 -degree component if $n \neq 0$. It remains to compute $\mathcal{H}_{0}(k)$. and $\mathcal{H}_{1}(k)$.

Clearly $\mathcal{H}_{0}(k)=k$. For $\mathcal{H}_{1}$, since $\mathfrak{B} / \mathfrak{B}_{<t o p} \cong k$ (notice $x y \in \mathfrak{B}_{\text {top }}$ has degree zero), the formula for $T^{\prime}$ is

$$
T^{\prime}(k)=k \oplus k x \oplus k y
$$

We have $\left(T^{\prime} k\right)_{0}=k=\left(T^{\prime} k\right)^{c o H}$ and $d B=B d=0$ in $T^{\prime} k$, so $\mathcal{H}_{1}(k)=\mathcal{H}_{0}\left(T^{\prime} k\right)=k$.

Remark 4.11. Notice the asymmetry in the gradings, $\mathcal{H}_{1}(k)=k$ but $\mathcal{H}_{-1}(k)=0$, as we can see from the general argument above, or compute directly:

$$
T(k)=\mathfrak{B} / k=k x \oplus k y \oplus k x y
$$

The degree zero component is $k x y$, but $d(x y) \neq 0$ (also $B(x y) \neq 0$ ), so $T(k)^{c o H}=0$ and

$$
\mathcal{H}_{-1}(k)=\mathcal{H}_{0}(T(k))=\frac{T(k)^{c o H}}{\Lambda \cdot T(k)}=0
$$




\section{5 (De)Categorification: computation of $K_{0}$}

\section{$5.1 K_{0}$ of exact and triangulated categories}

The main result of this section is Theorem 5.1, that gives a general presentation of $K_{0}\left(\underline{\mathfrak{m}}^{H}\right)$. We recall the main constructions:

If $\mathcal{A}$ is an exact category such that the isomorphism classes of objects is a set, then $K_{0}(\mathcal{A})$ is defined as the free abelian group on the set of isomorphism classes of objects module the relations

$$
[X]+[Z]=[Y]
$$

whenever there is a short exact sequence

$$
0 \rightarrow X \rightarrow Y \rightarrow Z \rightarrow 0
$$

We remark that $[X]+[Y]=[X \oplus Y]$ and, for $n \in \mathbb{N}, n[X]=\left[X^{n}\right]$, so, every element in $K_{0}(\mathcal{A})$ can be written in the form $[X]-[Y]$ for some objects $X, Y$ in $\mathcal{A}$. For triangulated categories, $K_{0}$ is defined similarly, taking the free abelian group on isomorphism classes of objects modulo the relations

$$
[X]+[Z]=[Y]
$$

whenever there is a triangle

$$
X \rightarrow Y \rightarrow Z \rightarrow T X
$$

By $K_{0}\left(\mathfrak{m}^{H}\right)$ we understand the K-theory of the category of finite dimensional $H$ comodules, that is an exact category with usual short exact sequences. We denote $K_{0}\left(\underline{\mathfrak{m}}^{H}\right)$ the K-theory of the stable category $\underline{\mathfrak{m}}^{H}$ as triangulated category.

Almost by definition, if $\mathcal{I}$ denotes the full subcategory of injective objects in $\mathfrak{m}^{H}$, there is a short exact exact sequence of categories

$$
0 \rightarrow \mathcal{I} \rightarrow \mathfrak{m}^{H} \rightarrow \underline{\mathfrak{m}}^{H} \rightarrow 0
$$

One could expect a general result in K-theory concluding a long exact sequence ending with

$$
K_{0}(\mathcal{I}) \rightarrow K_{0}\left(\mathfrak{m}^{H}\right) \rightarrow K_{0}(\underline{\mathfrak{m}})^{H} \rightarrow 0
$$

This is actually the case for short exact sequences of exact categories where the left hand side is also a Serre subcategory. Recall a Serre (sub)category is closed under quotients, subobjects and extensions. In our case, $\mathfrak{m}^{H}$ is an exact category but $\mathcal{I}$ is not a Serre subcategory in general. Also, $\underline{\mathfrak{m}}^{H}$ is not in general an exact category, it is triangulated, but the other two are not triangulated.

For exact sequences of Waldhausen categories there is also a long exact sequence in $K$-theory, both $\mathcal{I}$ and $\mathfrak{m}^{H}$ are Waldhausen categories, but it is not clear that $\underline{\mathfrak{m}}^{H}$ is so, in any case one should prove it. Instead, one can prove directly the following:

Theorem 5.1. The natural functors $\mathcal{I} \rightarrow \mathfrak{m}^{H}$ and $\mathfrak{m}^{H} \rightarrow \underline{\mathfrak{m}}^{H}$ induce an exact sequence

$$
K_{0}(\mathcal{I}) \rightarrow K_{0}\left(\mathfrak{m}^{H}\right) \rightarrow K_{0}\left(\underline{\mathfrak{m}}^{H}\right) \rightarrow 0
$$

In particular, the ring $K_{0}\left(\underline{\mathfrak{m}}^{H}\right)$ can be presented as the quotient of $K_{0}\left(\mathfrak{m}^{H}\right)$ by the ideal generated by injective objects. 
Proof. The functor $\mathfrak{m}^{H} \rightarrow \underline{\mathfrak{m}}^{H}$ is the identity on objects, so the induced map $K_{0}\left(\mathfrak{m}^{H}\right) \rightarrow$ $K_{0}\left(\underline{\mathfrak{m}}^{H}\right)$ is surjective. By definition of $\underline{\mathfrak{m}}^{H}$, the composition $\mathcal{I} \rightarrow \mathfrak{m}^{H} \rightarrow \underline{\mathfrak{m}}^{H}$ is zero, so the composition $K_{0}(\mathcal{I}) \rightarrow K_{0}\left(\mathfrak{m}^{H}\right) \rightarrow \overline{K_{0}}(\underline{\mathfrak{m}})^{H}$ is zero as well. Let us denote

$$
K_{0}\left(\mathfrak{m}^{H}\right) / K_{0}(\mathcal{I}):=\frac{K_{0}\left(\mathfrak{m}^{H}\right)}{\operatorname{Im}\left(K_{0}(\mathcal{I}) \rightarrow K_{0}\left(\mathfrak{m}^{H}\right)\right)}
$$

We have a surjective ring homomorphism

$$
K_{0}\left(\mathfrak{m}^{H}\right) / K_{0}(\mathcal{I}) \rightarrow K_{0}\left(\underline{\mathfrak{m}}^{H}\right)
$$

To see injectivity of this map we argue as follows: Assume $\omega=[M]-[N]$ in $K_{0}\left(\mathfrak{m}^{H}\right)$ goes to zero in $K_{0}\left(\underline{\mathfrak{m}}^{H}\right)$. From the short exact sequence

$$
0 \rightarrow N \rightarrow I(N) \rightarrow T N \rightarrow 0
$$

in $\mathfrak{m}^{H}$ with $I(N)$ injective, $[T N]=[I(N)]-[N]$ in $K_{0}\left(\mathfrak{m}^{H}\right)$, hence $[T N]=-[N]$ in $K_{0}\left(\mathfrak{m}^{H}\right) / K_{0}(\mathcal{I})$. So, we have

$$
\omega=[M]-[N]=[M \oplus T N] \operatorname{Mod} \mathcal{I}
$$

Eventually changing $\omega=[M]-[N]$ by $\omega^{\prime}:=[M \oplus T N]$, we can assume that, modulo $\mathcal{I}$, the element $\omega$ is equal to $[M]$ for some object $M$. Now if $[M]$ is zero in $K_{0}\left(\underline{\mathfrak{m}}^{H}\right)$, then there exists integers $n_{i}$ and triangles in the stable category

$$
X_{i} \rightarrow Y_{i} \rightarrow Z_{i} \rightarrow T X_{i}
$$

such that

$$
[M]=\sum_{i} m_{i}\left(\left[X_{i}\right]+\left[Z_{i}\right]-\left[Y_{i}\right]\right)
$$

But, using that direct sum of triangles is a triangle, for the positive $m_{i}$ 's we get

$$
\sum_{i} m_{i}\left(\left[X_{i}\right]+\left[Z_{i}\right]-\left[Y_{i}\right]\right)=\left[\oplus_{m_{i}>0} X_{i}^{m_{i}}\right]+\left[\oplus_{m_{i}>0} Z_{i}^{m_{i}}\right]-\left[\oplus_{m_{i}>0} Y_{i}^{m_{i}}\right]
$$

and similarly for the negative $m_{i}$ 's. From this, we may assume that there are two triangles $X_{i} \rightarrow Y_{i} \rightarrow Z_{i} \rightarrow T X_{i}, i=1,2$ such that

$$
[M]=\left(\left[X_{1}\right]+\left[Z_{1}\right]-\left[Y_{1}\right]\right)-\left(\left[X_{2}\right]+\left[Z_{2}\right]-\left[Y_{2}\right]\right)
$$

But because $X \stackrel{\text { id }}{\rightarrow} X \rightarrow 0 \rightarrow T X$ is a triangle, then so is $X \rightarrow 0 \rightarrow T X \rightarrow T X \rightarrow$, hence $[T X]=-[X]$ in $K_{0}\left(\underline{\mathfrak{m}}^{H}\right)$, and for $X \rightarrow Y \rightarrow Z \rightarrow T X$ a triangle, we have $T X \rightarrow T Y \rightarrow T Z \rightarrow T^{2} X$ is also a triangle and

$$
-([X]+[Z]-[Y])=[T X]+[T Z]-[T Y]
$$

So, we can conclude that there exists a single triangle $X \rightarrow Y \rightarrow Z \rightarrow$ such that

$$
[M]=[X]+[Z]-[Y]
$$


But we know (Lemma 1.10) that any triangle in the stable category $X \rightarrow Y \rightarrow Z \rightarrow T X$ is isomorphic, in the stable category, to a short exact sequence

$$
0 \rightarrow X^{\prime} \rightarrow Y^{\prime} \rightarrow Z^{\prime} \rightarrow 0
$$

Recall also that $X \cong X^{\prime}$ in $\underline{\mathfrak{m}}^{H}$ if and only if there exist injectives $I$ and $J$ such that $X \oplus I \cong X^{\prime} \oplus J$ in $\mathfrak{m}^{H}$. But Modulo $\mathcal{I}$, clearly $[X]=[X]+[I]=[X \oplus I]=\left[X^{\prime} \oplus J\right]=$ $\left[X^{\prime}\right]+[J]=\left[X^{\prime}\right]$. So, we finally get that

$$
[M]=\left[X^{\prime}\right]+\left[Z^{\prime}\right]-\left[Y^{\prime}\right] \operatorname{Mod} \mathcal{I}
$$

Hence, $[M]$ in $K_{0}\left(\mathfrak{m}^{H}\right)$ is zero $\operatorname{Mod} K_{0}(\mathcal{I})$.

Remark 5.2. It could be interesting to know if this is the last part of a long exact sequence for higher K-groups.

\section{$5.2 K_{0}$ and the coradical}

Let $H$ be a Hopf algebra and $H_{0}$ its coradical. Since $H_{0}$ is a subcoalgebra, every $H_{0^{-}}$ comodule is an $H$-comodule. Consider the category $\mathcal{A}=\mathfrak{m}^{H}$ and $\mathcal{B}=\mathfrak{m}^{H_{0}} ; \mathcal{B}$ is a non-empty full subcategory closed under taking subobjects, quotient objects, and finite products in $\mathcal{A}$. Also $\mathcal{B}$ is an abelian category and the inclusion functor $\mathcal{B} \rightarrow \mathcal{A}$ is exact, so Quillen's theorem gives.

Theorem 5.3. ([Q], Theorem 4. (Devissage)) Let $\mathcal{B}$ and $\mathcal{A}$ be as above. Suppose that every object $M$ of $\mathcal{A}$ has a finite filtration $0=M_{0} \subseteq M_{1} \subseteq \cdots \subseteq M_{n}=M$ such that $M_{j} / M_{j-1}$ is in $\mathcal{B}$ for each $j$. Then the inclusion $\mathcal{B} \rightarrow \mathcal{A}$ induces an isomorphism $K_{\bullet}(\mathcal{B}) \cong K_{\bullet}(\mathcal{A})$

If $M \in \mathcal{M}^{H}$ is a nonzero comodule, then its socle $\operatorname{soc}(M)$ is a nonzero subcomodule that is actually an $H_{0}$-comodule (see Exercise 3.1.2. of [DNR], page 117, its solution on page 140). If in addition $M$ is finite dimensional, considering $M / \operatorname{soc}(M)$ and induction in the dimension of $M$ one can easily define a finite filtration

$$
0=\operatorname{soc}(M) \subseteq M_{2} \subseteq \cdots \subseteq M_{n}=M
$$

such that $M_{j} / M_{j-1}=\operatorname{soc}\left(M_{j} / M_{j-1}\right)$, hence $M_{j} / M_{j-1} \in \mathfrak{m}^{H_{0}}$. Now Quillen's theorem implies the following:

Corollary 5.4. As abelian groups, $K_{0}\left(\mathfrak{m}^{H}\right) \cong K_{0}\left(\mathfrak{m}^{H_{0}}\right)$. If $H_{0}$ is a Hopf subalgebra then this isomorphism is also a ring isomorphism.

\subsection{Smash products}

Let $H=H_{0} \# \mathfrak{B}$ where $H_{0}$ is cosemisimple and $\mathfrak{B}$ a finite dimensional braided Hopf algebra in ${ }_{H_{0}} \mathcal{Y} \mathcal{D}^{H_{0}}$. For an element $M \in \mathcal{M}^{H}$, denote gr $M$ the associated graded with respect to the "socle filtration". Recall that the assignment $[M] \mapsto[\operatorname{gr} M]$ implements the isomorphism $K_{0}\left(\mathfrak{m}^{H}\right) \cong K_{0}\left(\mathfrak{m}^{H_{0}}\right)$. If $\left\{S_{i}: i \in I\right\}$ denote the set of (isomorphism classes of) simple objects in $\mathcal{M}^{H_{0}}$, then, for $M \in \mathfrak{m}^{H}$,

$$
\operatorname{gr} M \cong \oplus_{i \in I} S_{i}^{m_{i}}
$$


for uniques (and finite non zero) multiplicity integers $m_{i}=m_{i}(M)$. We define

$$
[M]_{H_{0}}:=\sum_{i} m_{i}\left[S_{i}\right]=[\operatorname{gr} M] \quad \in K_{0}\left(\mathfrak{m}^{H_{0}}\right)=\bigoplus_{i \in I} \mathbb{Z}\left[S_{i}\right]
$$

In particular $\mathfrak{B}$ is a finite dimensional $H$-comodule, so it makes sense

$$
[\mathfrak{B}]_{H_{0}} \in K_{0}\left(\mathfrak{m}^{H_{0}}\right)
$$

In the case $H_{0}=k[G]$ with $G$ a group, the isomorphism classes of simple comodules can be parametrized by $\{k g\}_{g \in G}$ and $k g \otimes k h \cong k g h$, so we identify $K_{0}\left(\mathfrak{m}^{k[G]}\right) \cong \mathbb{Z}[G]$. The main result of this section is the following:

Theorem 5.5. Let $G$ be a group and $H_{0}=k[G]$. Assume $H=H_{0} \# \mathfrak{B}$, with finite dimensional $\mathfrak{B}$. The assignment $[M] \mapsto[M]_{H_{0}}$ induces an isomorphism of rings

$$
K_{0}\left(\underline{\mathfrak{m}}^{H}\right) \cong \mathbb{Z}[G] /\left([\mathfrak{B}]_{H_{0}}\right)
$$

Proof. From Theorem 5.1 it follows that $K_{0}\left(\underline{\mathfrak{m}}^{H}\right) \cong K_{0}\left(\mathfrak{m}^{H}\right) / K_{0}(\mathcal{I})$. But from Corollary 5.4 we know

$$
\begin{gathered}
K_{0}\left(\mathfrak{m}^{H}\right) \cong K_{0}\left(\mathfrak{m}^{H_{0}}\right) \\
M \mapsto[\operatorname{gr} M]
\end{gathered}
$$

For $H_{0}=k[G]$ we also know $K_{0}\left(\mathfrak{m}^{k[G]}\right) \cong \mathbb{Z}[G]$. We need to identify $\mathcal{K}_{0}(\mathcal{I})$ inside $K_{0}\left(\mathfrak{m}^{H}\right) \cong \mathbb{Z}[G]$.

Recall that $\mathfrak{B}$ is injective and $\mathfrak{B}^{c o H}=k$. Now let $I$ be a finite dimensional indecomposable injective $H$-comodule. Because $I$ is indecomposable and injective, $\operatorname{soc}(I)$ is an indecomposable $H_{0}$-comodule (here, injectivity of $I$ is essential), hence simple and

$$
\operatorname{soc}(I) \cong k g
$$

for some $g \in G$. Clearly $\widetilde{I}:=I \otimes k g^{-1}$ is an injective indecomposable $H$-comodule and $(\widetilde{I})^{c o H}=k$. Since $\widetilde{I}$ is injective, there exist a dashed morphism in the diagram:

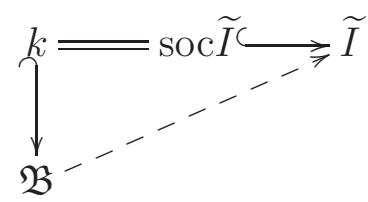

This map restricted to the socle is injective, so the map is injective and we have $\operatorname{dim} \mathfrak{B} \leq$ $\operatorname{dim} \widetilde{I}$. But $\mathfrak{B}$ is injective, so the same argument in the opposite direction gives $\operatorname{dim} \widetilde{\mathcal{I}} \leq$ $\operatorname{dim} \mathfrak{B}$ and so $B \cong \widetilde{I}$. In other words,

$$
I \cong \mathfrak{B} \otimes k g
$$

for some $g \in G$. We can conclude that if $I$ is finite dimensional injective (non necessarily indecomposable) $H$-comodule, then there exists integers $\left\{m_{g}: g \in G\right\}$ with

$$
I \cong \bigoplus_{g \in G} m_{g} \mathfrak{B} \otimes k g
$$

That is, $\operatorname{Im}\left(K_{0}(\mathcal{I}) \rightarrow K_{0}\left(\mathfrak{m}^{H}\right)\right)$ is the ideal generated by $[\mathfrak{B}]$. 


\subsection{Examples}

The first two examples are well-known:

1. $H=k[\mathbb{Z}] \# k[x] / x^{2}:$

$$
K_{0}\left(\underline{\mathfrak{m}}^{H}\right) \cong K_{0}(k[\mathbb{Z}]) /\left\langle k[x] / x^{2}\right\rangle \cong \mathbb{Z}\left[z^{ \pm 1}\right] /(1+z) \cong \mathbb{Z}
$$

2. (Khovanov) $H=k[\mathbb{Z}] \# k[x] / x^{N}$ :

$$
K_{0}\left(\underline{\mathfrak{m}}^{H}\right) \cong K_{0}(k[\mathbb{Z}]) /\left\langle k[x] / x^{N}\right\rangle \cong \mathbb{Z}\left[z^{ \pm 1}\right] /\left(1+z+\cdots z^{N-1}\right)
$$

If $N$ is is a prime $p$ then $K_{0}\left(\underline{\mathfrak{m}}^{H}\right) \cong \mathbb{Z}\left[\xi_{p}\right]$.

3. $H=k[\mathbb{Z}] \# \Lambda(x, y)$ where $|x|=1, \mid x=-1$, then

$$
\Lambda(x, y)=k \oplus k x \oplus k y \oplus k x y
$$

hence $[\operatorname{gr} \Lambda(x, y)]=1+z+z^{-1}+1=z^{-1}+2+z=z^{-1}(1+z)^{2}$ and so

$$
K_{0}\left(\underline{\mathfrak{m}}^{H}\right) \cong \mathbb{Z}\left[z^{ \pm 1}\right] /(1+z)^{2}=\mathbb{Z}[z] /(1+z)^{2} \cong \mathbb{Z}[t] / t^{2}
$$

4. If $N_{1}, \ldots, N_{k} \in \mathbb{N}$, for $1 \leq i<j \leq k$, a list of nonzero scalars $q_{i j} \in k^{\times}$is given, then define $H$ as the algebra generated by $x_{1}, \ldots, x_{k}, g_{1}^{ \pm 1}, \ldots, g_{k}^{ \pm 1}$ with relations

$$
\begin{aligned}
g_{i} g_{j} & =g_{j} g_{i} & & (\forall i, j) \\
x_{i} x_{j} & =q_{i j} x_{j} x_{i} & & (i<j) \\
x_{i} g_{j} & =q_{i j} g_{j} x_{i} & & (i<j) \\
g_{i} x_{j} & =q_{i j} x_{j} g_{i} & & (i<j) \\
x_{i}^{N_{i}} & =0 & &
\end{aligned}
$$

It is a Hopf algebra with comultiplication given by

$$
\begin{gathered}
\Delta g_{i}=g_{i} \otimes g_{i} \\
\Delta x_{i}=x_{i} \otimes g_{i}+1 \otimes x_{i}
\end{gathered}
$$

Then $H$ is a Hopf algebra of the form $k\left[\mathbb{Z}^{k}\right] \# \mathfrak{B}$. The algebra $\mathfrak{B}$ has monomial basis $\left\{x_{1}^{n_{1}} \cdots x_{k}^{n_{k}}, 0 \leq n_{i}<N_{i}\right\}$, so, writing $\mathbb{Z}\left[\mathbb{Z}^{k}\right]=\mathbb{Z}\left[z_{1}^{ \pm 1}, \ldots, z_{k}^{ \pm 1}\right]$,

$$
[\mathfrak{B}]_{H_{0}}=\prod_{i=1}^{k}\left(1+z_{i}+z_{i}^{2}+\cdots+z_{i}^{N_{i}-1}\right)
$$

Hence,

$$
K_{0}\left(\underline{\mathfrak{m}}^{H}\right) \cong \mathbb{Z}\left[z_{1}^{ \pm 1}, \cdots, z_{k}^{ \pm 1}\right] / \prod_{i=1}^{k}\left(1+z_{i}+\cdots z_{i}^{N_{i}-1}\right)
$$

Remark 5.6. It would be interesting to compute $K_{0}\left(H_{0} \# \mathfrak{B}\right)$ for some non pointed cosemisimple $H_{0}$, for instance, $H_{0}=\mathcal{O}(G)$ with $G$ non abelian reductive affine group. 


\section{$6 H$-comodule algebras and the category ${ }_{A} \mathcal{M}^{H}$}

An $H$-comodule algebra $A$ is a $k$-algebra $A$ together with an $H$-comodule structure such that the multiplication map

$$
A \otimes A \rightarrow A
$$

and the unit

$$
k \rightarrow A
$$

are $H$-colinear. Usual examples are:

- $H=k[G]$ : comodule algebra = G-graded algebra.

- $G$ finite, $H=k^{G}$ : comodule algebra = algebra with a $G$-action by ring homomorphisms.

- $G$ affine group, $H=\mathcal{O}(G)$ : comodule algebra = algebra with a rational $G$-action.

- $H=U \mathfrak{g}$, comodule algebra =algebra with a $\mathfrak{g}$-action acting by derivations.

For our purpose, the motivating example is $H=k[\mathbb{Z}] \# k[x] / x^{2}$. In this case, an $H$-comodule algebra $=$ d.g. algebra.

Also, if $H$ is any Hopf algebra and $A$ is any algebra, then $A$ viewed as trivial $H$ comodule is an $H$-comodule algebra.

The main fact for our interest is the following:

$$
M \in{ }_{A} \mathcal{M}^{H}, V \in \mathcal{M}^{H} \Rightarrow M \otimes V \in{ }_{A} \mathcal{M}^{H}
$$

where $A$-module structure in $M \otimes V$ is the one coming from $M$ and the $H$-comodule structure is the diagonal one. Moreover, if $M$ is finitely generated as $A$-module and $V$ is finite dimensional, then $A \otimes V$ is finitely generated as $A$-module. In this way, the subcategory of ${ }_{A} \mathcal{M}^{H}$ consisting on $A$-finitely generated modules, denoted by ${ }_{A} \mathfrak{m}^{H}$, is naturally a module over the category $\mathfrak{m}^{H}$. Following $[\mathrm{K}]$, we consider the restriction functor

$$
{ }_{A} \mathcal{M}^{H} \rightarrow \mathcal{M}^{H}
$$

and define $M \in{ }_{A} \mathcal{M}^{H} \rightarrow \mathcal{M}^{H}$ to be acyclic (or $H$-acyclic to emphasize the role of $H$ ) if $M$ is injective as $H$-comodule. In other words, if $M \cong 0$ in $\underline{\mathcal{M}}^{H}$. A map $f: M \rightarrow N$ is called quasi-isomorphimsm (qis) if $f$ becomes an isomorphism in $\underline{\mathcal{M}}^{H}$. Denote $\mathcal{I}_{A}$ the class of objects in ${ }_{A} \mathcal{M}^{H}$ that are injective as $H$-comodules.

Example 6.1. Let $M \in{ }_{A} \mathcal{M}^{H}$ be an arbitrary object and $I \in \mathcal{M}^{H}$ an injective $H$ comodule. In virtue of Lemma 2.14, $M \otimes I \in \mathcal{I}_{A}$.

If $M, N \in{ }_{A} \mathcal{M}^{H}$, denote $\mathcal{I}_{A}(M, N)$ the set of maps that factors through an object in $\mathcal{I}_{A}$. The stable category - or the $H$-derived category- , denoted by ${ }_{A} \mathcal{M}^{H}$ and also by $\mathcal{D}_{H}(A)$, is defined as the category with same objects as ${ }_{A} \mathcal{M}^{H}$ but morphism

$$
\operatorname{Hom}_{\mathcal{D}_{H}(A)}(M, N):=\frac{\operatorname{Hom}_{A}^{H}(M, N)}{\mathcal{I}_{A}(M, N)}
$$


The subcategory of $\mathcal{D}_{H}(A)$ whose objects are in ${ }_{A} \mathfrak{m}^{H}$ (i.e. are finitely generated as $A$-modules) is denoted by $\mathcal{D}_{H}^{c}(A)$.

Recall that if $E=E(k)$ is the injective hull of $k, E$ is a finite dimensional injective $H$-comodule (because $H$ is co-Frobenius), and for any $M \in{ }_{A} \mathcal{M}^{H}$, then

$$
M \rightarrow M \otimes E
$$

is an embedding of $M$ into an acyclic object in ${ }_{A} \mathcal{M}^{H}$. If $P:=P(k)$ is a (finite dimensional) projective cover of $k$, then

$$
M \otimes P \rightarrow M
$$

is an epimorphism from an $H$-acyclic object in ${ }_{A} \mathcal{M}^{H}$ into $M$. If $M$ is finitely generated as $A$-module, then so is $M \otimes E$ and $M \otimes P$. The definition of $T M$, of $T^{\prime} M$ and of the mapping cone of objects and maps in ${ }_{A} \mathcal{M}^{H}$ (resp. in ${ }_{A} \mathfrak{m}^{H}$ ) actually gives objects in ${ }_{A} \mathcal{M}^{H}$ (resp. in ${ }_{A} \mathfrak{m}^{H}$ ). One can easily see that all constructions and proof's of Happel's Theorem 2.6 in [Ha], when starting with objects in in ${ }_{A} \mathcal{M}^{H}$ (resp. in ${ }_{A} \mathfrak{m}^{H}$ ) always stay in ${ }_{A} \mathcal{M}^{H}$ (resp. in ${ }_{A} \mathfrak{m}^{H}$ ). So $\mathcal{D}_{H}(A)$ and $\mathcal{D}_{H}^{c}(A)$ are triangulated categories, and by Example 6.1, they are modules over $\underline{\mathcal{M}}^{H}$ and $\underline{\mathfrak{m}}^{H}$ respectively.

Remark 6.2. $K_{0}\left(\mathcal{D}_{H}^{c}(A)\right)$ is a module over the ring $K_{0}\left(\underline{\mathfrak{m}}^{H}\right)$.

Example 6.3. If $A=k$ then $\mathcal{D}_{H}(k)=\underline{\mathcal{M}}^{H}$ and $\mathcal{D}_{H}^{c}(k)=\underline{\mathfrak{m}}^{H}$.

Example 6.4. if $H=k[\mathbb{Z}] \# k[x] / x^{2}$ and $A$ is an ordinary algebra viewed as trivial $H$ comodule algebra then $\mathcal{D}_{H}(A)=\mathcal{D}(A)$, the (unbounded) derived category of $A$.

Example 6.5. If $A$ is a semisimple Hopf algebra and $H$ is a co-Frobenius Hopf algebra, we view $A$ as trivial $H$-comodule algebra, then

$$
{ }_{A} \mathcal{M}^{H} \cong \mathcal{M}^{A^{*} \otimes H}
$$

Since $A$ is semisimple, $A^{*}$ is co-semisimple and $A^{*} \otimes H$ is co-Frobenius. In this case we have $\mathcal{D}_{H}(A)=\underline{\mathfrak{m}}^{\left(A^{*} \otimes H\right)}$. Also if $H=k[G] \# \mathfrak{B}$ as in Section 5.3 then $K_{0}\left(\mathcal{D}_{H}(A)\right)=$ $K_{0}\left(\underline{\mathfrak{m}}^{\left.A^{*} \otimes k[G] \# \mathfrak{B}\right)}\right.$ is a quotient of $K_{0}\left(\mathfrak{m}^{A^{*} \otimes k[G]}\right)$. Assuming $k$ algebraically closed, every simple corepresentation of the tensor product $A^{*} \otimes k[G]$ is given by the tensor product of a simple $A^{*}$-comodule and a simple $k[G]$-comodule, hence $K_{0}\left(\mathfrak{m}^{A^{*} \otimes k[G]}\right)=$ $K_{0}\left(\mathfrak{m}^{A^{*}}\right) \otimes_{\mathbb{Z}} \mathbb{Z}[G]=K_{0}(A) \otimes_{\mathbb{Z}} \mathbb{Z}[G]$.

\section{Enriched Hom}

If $M, N \in{ }_{A} \mathcal{M}^{H}$, there are several Hom spaces that one can consider. We begin with the discussion for d.g. A-modules:

If $M$ and $N$ are d.g. A-modules, then one may consider

- Chain maps: $\operatorname{Hom}_{A}^{H}(M, N)=$ maps preserving degree and commuting with the differential.

- Chain maps up to homotopy: $\operatorname{Hom}_{A}^{H}(M, N) / \sim$, where $f \sim g$ if $f-g=d h+h d$ for some degree $+1 A$-linear map $h$. 
- The HOM complex: $\operatorname{HOM}_{A}(M, N)=\oplus_{n \in \mathbb{Z}} \operatorname{HOM}_{A}(M, N)_{n}$ where $\operatorname{HOM}_{A}(M, N)_{n}=$ $A$-linear maps of degree $n$. If $A$ is concentrated in degree zero (i.e. $A$ is a trivial $k[\mathbb{Z}] \# k[x] / x^{2}$-comodule) then $\operatorname{HOM}_{A}(M, N)_{n}=\prod_{q \in \mathbb{Z}} \operatorname{Hom}_{A}\left(M_{q}, N_{q+n}\right)$

- Morphisms in the derived category: $\operatorname{Hom}_{\mathcal{D}_{H}(A)}(M, N)$.

In general $\operatorname{HOM}_{A}(M, N)$ is different from $\operatorname{Hom}_{A}(M, N)$. Assume for simplicity $A$ is an ordinary alegbra (i.e. d.g. algebra concentrated in degree zero), if $M$ and $N$ have infinite nonzero degrees, then

$$
\operatorname{Hom}_{A}(M, N)=\operatorname{Hom}_{A}\left(\oplus_{p} M_{p}, \oplus_{q} N_{q}\right) \varsubsetneqq \bigoplus_{n}\left(\prod_{q} \operatorname{Hom}_{A}\left(M_{q}, N_{q+n}\right)\right)
$$

For instance, if $M=\oplus_{n} A[n]$ and $N=A$, then

$$
\operatorname{Hom}_{A}\left(\oplus_{n} A[n], A\right) \nsucc \oplus_{n} \operatorname{Hom}_{A}(A[n], A)
$$

Nevertheles, the set of chain maps agree with $B^{0}\left(\operatorname{HOM}_{A}(M, N)\right)$ and the set of chain maps up to homotopy is the same as $H_{0}\left(\operatorname{HOM}_{A}(M, N)\right)$.

For general co-Frobenius Hopf alegbras (i.e. not necesariily finite dimensional ones) one has the same "problems" but also analogous solutions. First of all, if $H$ is a (not finite dimensional) Hopf algebra, $A$ an $H$-comodule algebra and $M, N \in{ }_{A} \mathcal{M}^{H}$, then $\operatorname{Hom}_{A}(M, N)$ is not an $H$-comodule in general. For instance, if $A=k=N$ and $M=H$, then $H^{*}$ is a not rational $H^{*}$-module, so it is not an $H$-comodule. In this way, if one consider

$$
\operatorname{Hom}_{A}(M, N)
$$

it is not expectable to have an object in $\mathcal{M}^{H}$.

It is not clear to the author how to get an object in $\mathcal{M}^{H}$ analogous to $\mathrm{HOM}_{A}$ (maybe $\operatorname{HOM}_{A}(M, N):=\lim _{\rightarrow \mu} \operatorname{Hom}_{A}\left(M_{\mu}, N\right)$, where $M_{\mu}$ runs over all $A$-finitely generated subobjects?). To have an object $\operatorname{HOM}_{A}(M, N) \in \mathcal{M}^{H}$ would provide the notion of map up to homotopy just by taking $H_{0}$. Nevertheles, we have the following

Proposition 6.6. $\operatorname{Hom}_{A}(M, N)$ is a (left) $H^{*}$-module and the definition of $\mathcal{H}_{0}^{H}$ can be naturally extenbded to $H^{*}$-modules.

Proof. The first statement is probably well-known, for completenes we exhibit the proof: First recall that if $K$ is a finite dimensional Hopf algebra and $M, N$ are $K$ modules (e.g. $K=H^{*}$ if $H$ is finite dimensional and $M, N \in \mathcal{M}^{H}$ ) then the standard action of an element $x \in K$ in a map $f$, acting on an alement $m \in M$ is given by

$$
(x \cdot f)(m):=x_{1} f\left(S\left(x_{2}\right) \cdot m\right)
$$

If $K=H^{*}$ and $M, N \in \mathcal{M}^{H}$ then the above formula is

$$
\begin{gathered}
(x \cdot f)(m)=x_{1} \cdot f\left(\left(S\left(x_{2}\right)\left(m_{1}\right)\right) m_{0}\right)=x_{2}\left(S\left(m_{1}\right)\right) x_{1} \cdot f\left(m_{0}\right)=x_{1}\left(f\left(m_{0}\right)_{1}\right) x_{2}\left(S\left(m_{1}\right)\right) f\left(m_{0}\right)_{0} \\
=x\left(f\left(m_{0}\right)_{1} S\left(m_{1}\right)\right) f\left(m_{0}\right)_{0}
\end{gathered}
$$


and the last term in the equality make sense for $x \in H^{*}$, independently on the dimension of $H$, so one defines the $H^{*}$-action of $x \in H^{*}$ on $f: M \rightarrow N$ via

$$
(x \cdot f)(m):=x\left(f\left(m_{0}\right)_{1} S\left(m_{1}\right)\right) f\left(m_{0}\right)_{0}
$$

In other words,

$$
x \cdot f=\left(1 \otimes m_{H}^{*}(x)\right)\left(\rho_{N} \otimes 1\right)(f \otimes S) \rho_{M}
$$

One can proof by standard diagramatic methods that this is an action, and $f$ is $H$ colinear (if and only if it is $H^{*}$-linear) if and only if

$$
x \cdot f=\epsilon(x) f=x(1) f \forall x \in H^{*}
$$

Concering the second statement, if $W$ is an $H^{*}$-module, one may define

$$
W^{H^{*}}=\{w \in W: x \cdot w=x(1) w\} \cong \operatorname{Hom}_{H}(k, W)
$$

If $W$ is a right $H$-comodule then it is clear that $W^{c o H}=\{w: \rho(w)=w \otimes 1\}=W^{H^{*}}$, so one can extend the definition of $\mathcal{H}_{0}^{H}$ on $H^{*} \mathcal{M}$ simply by

$$
\mathcal{H}_{0}^{H}(W):=\frac{W^{H^{*}}}{\Lambda \cdot W}
$$

If $W=\operatorname{Hom}_{A}(M, N)$ then $W^{H^{*}}=A$ linear and $H^{*}$-linear maps $=\operatorname{Hom}_{A}^{H}(M, N)$, and a definition of "chain maps up to homotopy" is available definig

$$
\mathcal{H}_{0}^{H}\left(\operatorname{Hom}_{A}(M, N)\right)=\frac{\operatorname{Hom}_{A}^{H}(M, N)}{\Lambda \cdot \operatorname{Hom}_{A}(M, N)}
$$

This recover the definition given in [Qi1] for finite dimensional Hopf algebras and when $M$ and $N$ are $\mathbb{Z}$-graded vector spaces, but we emphasizes that this definition makes sense in full generality for $H$ a co-Frobenius algebra (whose coradical is not necesarily finite over $k[\mathbb{Z}])$.

A warning on the notation in [Qi1], we call $\mathcal{H}_{0}^{H}$ what he calls $\mathcal{H}$ in the ungraded case. He defines $\mathcal{H}_{n}$ only in the graded case but using the degree shifting, and not the triangulated structure, so $\mathcal{H}_{n}$ in [Qi1] is different from our $\mathcal{H}_{n}^{H}$.

\section{References}

[A] N. Andruskiewitsch: An Introduction to Nichols Algebras. In Quantization, Geometry and Noncommutative Structures in Mathematics and Physics. Mathematical Physics Studies, Springer; Alexander Cardona, Pedro Morales, Hernn Ocampo, Sylvie Paycha, Andrs Reyes, eds. pp. 135-195 (2017).

[ACE] N. Andruskiewitsch, J. Cuadra, P. Etingof: On two finiteness conditions for Hopf algebras with nonzero integral, Ann. Sc. Norm. Super. Pisa, Cl. Sci. (5) Vol. XIV (2015), 1-40. 
[AC] N. Andruskiewitsch, J. Cuadra: On the structure of (co-Frobenius) Hopf algebras Journal of Noncommutative Geometry. Volume 7, Issue 1, pp. 83104 (2013).

[AD] N. Andruskiewitsch, S. Dascalescu: Co-Frobenius Hopf algebras and the coradical filtration. Math. Z. 243 (2003), 145-154.

[DNR] S. Dascalescu, C. Nastasescu, S. Raianu: Hopf algebras. An introduction. P. and App. Math., Marcel Dekker. 235. New York, NY: Marcel Dekker. ix, 401 p. (2001).

[Ha] D. Happel, Triangulated categories in the representation of finite dimensional algebras, London Mathematical Society Lecture Note Series, 1988.

[K] M. Khovanov: Hopfological algebra and categorification at a root of unity: the first step, J. Knot Theory and its Ramifications 25, No. 3, 26 p. (2016).

[N] A. Neeman, The Grothendieck duality theorem via Bousfields techniques and Brown representability, J. Amer. Math. Soc. 9 (1996), 205-236.

[P] B. Pareigis, A Non-Commutative Non-Cocommutative Hopf Algebra in Nature , J. of Alg. 70, 356-374 (1981).

[Qi1] You Qi, Hopfological Algebra, Compositio Mathematica 150(01): 1-45 (2014).

[Qi2] You Qi, Morphism spaces in stable categories of Frobenius algebras, Communications in Algebra 2019, https:/ / doi.org/10.1080/00927872.2018.1555835

[Q] D. Quillen, Higher algebraic K-theory. I. In: Algebraic K-theory, I: Higher K-theories (Proc. Conf., Battelle Memorial Inst., Seattle, Wash., 1972). Lecture Notes in Math., vol. 341, pp. 85147. Springer, Berlin (1973)

[T] M. Takeuchi, Survey of braided Hopf algebras. New trends in Hopf algebra theory (La Falda, 1999), 301-323, Contemp. Math., 267, A.M.S., Providence, RI, 2000. 